\title{
Volume and topology of bounded and closed hyperbolic 3-manifolds
}

\author{
Jason DeBlois and Peter B. Shalen
}

Let $N$ be a compact, orientable hyperbolic 3-manifold with $\partial N$ a connected totally geodesic surface of genus 2. If $N$ has Heegaard genus at least 5 , then its volume is greater than 6.89 . The proof of this result uses the following dichotomy: either the shortest return path (defined by Kojima-Miyamoto) of $N$ is long, or $N$ has an embedded codimension- 0 submanifold $X$ with incompressible boundary $T \sqcup \partial N$, where $T$ is the frontier of $X$ in $N$, which is not a book of $I$-bundles. As an application of this result, we show that if $M$ is a closed, orientable hyperbolic 3-manifold with $\operatorname{dim}_{\mathbb{Z}_{2}} H_{1}\left(M ; \mathbb{Z}_{2}\right) \geq 5$, and if the cup product map $H^{1}\left(M ; \mathbb{Z}_{2}\right) \otimes$ $H^{1}\left(M ; \mathbb{Z}_{2}\right) \rightarrow H^{2}\left(M ; \mathbb{Z}_{2}\right)$ has image of dimension at most one, then $M$ has volume greater than 3.44 .

\section{Introduction}

The results of this paper support an old theme in the study of hyperbolic 3 -manifolds, that the volume of a hyperbolic 3-manifold increases with its topological complexity. The first main result, Theorem 1.1 below, reflects this theme in the context of hyperbolic manifolds with totally geodesic boundary. We denote the Heegaard genus of a 3-manifold $N$ by $\operatorname{Hg}(N)$.

Theorem 1.1. Let $N$ be a compact, orientable hyperbolic 3-manifold with $\partial N$ a connected totally geodesic surface of genus 2 . If $\operatorname{Hg}(N) \geq 5$, then $N$ has volume greater than 6.89 .

Our second main result concerns closed manifolds:

Theorem 1.2. Let $M$ be a closed, orientable hyperbolic 3-manifold with

$$
\operatorname{dim}_{\mathbb{Z}_{2}} H_{1}\left(M ; \mathbb{Z}_{2}\right) \geq 5
$$

and suppose that the cup product map $H^{1}\left(M ; \mathbb{Z}_{2}\right) \otimes H^{1}\left(M ; \mathbb{Z}_{2}\right) \rightarrow H^{2}(M$; $\mathbb{Z}_{2}$ ) has image of dimension at most one. Then $M$ has volume greater than 3.44 . 
Theorem 1.1 builds on work by Kojima and Miyamoto. Miyamoto proved that the minimal-volume compact hyperbolic 3-manifolds with totally geodesic boundary of genus $g$ decompose into $g$ regular truncated tetrahedra, each with dihedral angle $\pi / 3 g$ [18, Theorem 5.4]. Their volumes increase with $g$, taking values $6.452 \ldots$ for $g=2$ and $10.428 \ldots$ for $g=3$. Miyamoto's theorem implies in particular that the minimal volume compact hyperbolic manifolds with totally geodesic boundary of genus $g$ have Heegaard genus equal to $g+1$.

Prior to the work of Miyamoto, Kojima-Miyamoto established the universal lower bound of $6.452 \ldots$ for the volume of compact hyperbolic 3manifolds with totally geodesic boundary and described the minimal-volume examples [17]. In fact, their result is slightly stronger: if $N$ is not "simple," then $\operatorname{vol}(N)>6.47$. In the terminology of [17], a hyperbolic manifold with geodesic boundary is simple if it admits a decomposition into truncated polyhedra with one internal edge. Such manifolds are classified in [17, Lemma $2.2]$, and include those with minimal volume.

Theorem 1.1 can be regarded as an extension of Kojima-Miyamoto's theorem. Experimental results of Frigerio et al. [12] suggest that the next smallest manifolds with geodesic boundary after those of minimal volume have volume greater than 7.1, so it is likely that Theorem 1.1 is not close to sharp. Nonetheless it seems to be the only result of its kind in the literature.

Theorem 1.2 will be deduced by combining Theorem 1.1 with results from $[6,8]$. The transition from these results to Theorem 1.2 involves two other results, Theorems 1.3 and 1.4 below, which are of independent interest.

Theorem 1.3. Let $M$ be a closed, orientable hyperbolic 3-manifold containing a closed, connected incompressible surface of genus 2 or 3 , and suppose that $\operatorname{Hg}(M) \geq 8$. Then $M$ has volume greater than 6.89 .

Theorem 1.3 is analogous to [6, Theorem 6.5], and follows in a similar way: we apply Theorem 1.1 and the results of Miyamoto and KojimaMiyamoto discussed above, using work of Agol et al. [2], to the output of the topological theorem below. The notation in the statement is taken from [6]. In particular, below and in the remainder of this paper, we will use the term "simple" as it is defined in [6, Definitions 1.1], which differs from its usage in [17] mentioned above. We also recall the definitions of $M \backslash \backslash S$ from the first sentence of [6], and "kish" from Definition 1.1 there.

Theorem 1.4. Suppose that $M$ is a closed, simple 3-manifold which contains a connected closed incompressible surface of genus 2 or 3 , and that 
$\operatorname{Hg}(M) \geq 8$. Then $M$ contains a connected closed incompressible surface $S$ of genus at most 4 , such that either $\bar{\chi}(\operatorname{kish}(M \backslash \backslash S)) \geq 2$, or $S$ is separating and $M \backslash \backslash S$ has an acylindrical component $N$ with $\operatorname{Hg}(N) \geq 7$.

Theorem 1.4 follows by application of [6, Theorem 5.8] jointly with [6, Theorem 3.2]. It is the analog of [6, Corollary 5.9] for manifolds possessing an incompressible surface of genus 3 .

The proof of Theorem 1.2 follows the outline of [6, Theorem 6.8]. In place of the results concerning 3-free groups used in [6], the proof uses results of [8] concerning 4-free groups, and Theorem 1.3 above replaces [6, Theorem 6.5].

All the theorems stated above are proved in Section 7 . Sections $2-6$ constitute preparation for the proof of Theorem 1.1. We introduce return paths, defined by Kojima [16], and $(i, j, k)$ hexagons in Section 2. (The analysis of $(i, j, k)$ hexagons is a crucial element of [17], but we have borrowed our notation for them from Gabai et al. [13] paper, which is set in a different context.) Lemmas 2.7 and 2.8, which are due to Kojima-Miyamoto [17], respectively, give an absolute lower bound on $\ell_{1}$, and a lower bound for $\ell_{2}$ in terms of $\ell_{1}$. Lemma 2.9 refines Lemma 2.8, giving a bound for $\ell_{2}$ which improves that of [17] when $\ell_{1}$ is in a certain interval.

Section 3 describes Kojima-Miyamoto's volume bounds. The main result rigorously establishes a lower bound which is apparent from inspection of [17, Graph 4.1].

Proposition 3.7. Let $N$ be a hyperbolic 3-manifold with $\partial N$ connected, totally geodesic and of genus 2 , satisfying $\cosh \ell_{1} \geq 1.215$. Then $N$ has volume greater than 6.89 .

Also in Section 3, Proposition 3.9 shows that any manifold with no $(1,1,1)$ hexagon has a shortest return path satisfying $\cosh \ell_{1} \geq 1.215$, thus has volume greater than 6.89 by the above. In the remaining sections, we explore the topological consequences of the presence of a $(1,1,1)$ hexagon in $\widetilde{N}$, when $\cosh \ell_{1} \leq 1.215$. In Section 6 , we show that under these circumstances, $N$ contains a submanifold $X$ which is a nondegenerate trimonic manifold relative to $\partial N$ (see Definition 5.7).

Section 4 develops results from the theory of books of $I$-bundles (which were introduced in [1]) that we use in Section 5. There we introduce trimonic manifolds and prove, in Proposition 5.14, that such a manifold $X$ does not have the structure of a book of $I$-bundles. It follows that $X$ has kishkes (or guts, cf. [2]) with negative Euler characteristic. Using volume bounds due to Agol-Storm-Thurston, we obtain the following result. 
Theorem 7.4. Let $N$ be a compact, orientable hyperbolic 3-manifold with $\partial N$ a connected totally geodesic surface of genus 2 . If $\cosh \ell_{1} \leq 1.215$ and there is a $(1,1,1)$ hexagon in $\widetilde{N}$, then $\operatorname{Hg}(N) \leq 4$ or $\operatorname{vol}(N)>7.32$.

Together with the results of Section 3, this implies Theorem 1.1.

\section{Geometric preliminaries}

Suppose $N$ is a hyperbolic 3-manifold with totally geodesic boundary. Its universal cover $\widetilde{N}$ may be identified with a convex subset of $\mathbb{H}^{3}$ bounded by a collection of geodesic hyperplanes. The following terminology was introduced in [16] and used extensively in [17]; we will use it here as well.

Definition 2.1. Let $N$ be a hyperbolic 3-manifold with totally geodesic boundary, and let $\widetilde{N} \subset \mathbb{H}^{3}$ be its universal cover. A short cut in $\widetilde{N}$ is a geodesic arc joining the closest points of two distinct components of $\partial \widetilde{N}$. A return path in $N$ is the projection of a short cut under the universal covering map.

It is an easy consequence of the definitions that each return path is a homotopically nontrivial geodesic arc properly immersed in $N$, perpendicular to $\partial N$ at each of its endpoints. Corollary 3.3 of [16] asserts that for a fixed hyperbolic manifold $N$ with geodesic boundary and $K \in \mathbb{R}$, there are only finitely many return paths in $N$ with length less than $K$. Thus the collection of return paths may be enumerated as $\left\{\lambda_{1}, \lambda_{2}, \ldots\right\}$, where for each $i \in \mathbb{N}$, the length of $\lambda_{i}$ is less than or equal to the length of $\lambda_{i+1}$. Fixing such an arrangement, we will denote by $\ell_{i}$ the length of $\lambda_{i}$.

It will prove important to understand the distance in $\partial N$, properly interpreted, between endpoints of return paths of $N$.

Definition 2.2. Let $N$ be a compact hyperbolic 3-manifold with connected totally geodesic boundary, and suppose $\lambda$ is a short cut in $\widetilde{N}$ projecting to $\lambda_{i}$. Fix an endpoint $x$ of $\lambda$, and let $\Pi$ be the component of $\partial \widetilde{N}$ containing $x$. For $j \in \mathbb{N}$ define $d_{i j}$ to be the minimum, taken over all short cuts $\lambda^{\prime}$ projecting to $\lambda_{j}$ such that $\lambda^{\prime}$ has an endpoint $y \in \Pi$ and $\lambda^{\prime} \neq \lambda$, of $d(x, y)$.

The requirement above that $\lambda^{\prime}$ be distinct from $\lambda$ ensures that $d_{i i}>0$. In general, $d_{i j}$ is the length of the shortest geodesic arc in $\partial N$ joining an endpoint of $\lambda_{i}$ to an endpoint of $\lambda_{j}$. 
A crucial tool for understanding the relationships between the lengths $\ell_{i}$ and distances $d_{i j}$ is a class of totally geodesic hexagons in $\widetilde{N}$ which have short cuts as edges. The two lemmas below describe the relevant hexagons.

Lemma 2.3. Suppose that $\Pi_{1}, \Pi_{2}$ and $\Pi_{3}$ are mutually disjoint geodesic planes in $\mathbb{H}^{3}$. For each two-element subset $\{i, j\}$ of $\{1,2,3\}$, let $\lambda_{i j}$ denote the common perpendicular to $\Pi_{i}$ and $\Pi_{j}$. Then $\lambda_{12}, \lambda_{13}$ and $\lambda_{23}$ lie in a common plane $\Pi$.

Proof. We may assume that the three lines $\lambda_{12}, \lambda_{13}$ and $\lambda_{23}$ do not all coincide, and so by symmetry we may assume that $\lambda_{12} \neq \lambda_{13}$. For $i=2,3$ the line $\lambda_{1 i}$ meets $\Pi_{1}$ orthogonally at some point $p_{i}$. Let $L \subset \Pi_{1}$ denote the line joining $p_{2}$ and $p_{3}$, and let $\Sigma$ denote the plane which meets $\Pi_{1}$ perpendicularly along $L$. It is clear that $\Sigma$ contains $\lambda_{12}$ and $\lambda_{13}$. This implies that $\Sigma$ meets the planes $\Pi_{2}$ and $\Pi_{3}$ perpendicularly. For $i=2,3$, let $X_{i}$ denote the line $\Pi_{i} \cap \Sigma$. Since $\Pi_{2} \cap \Pi_{3}=\emptyset$, the lines $X_{2}, X_{3} \subset \Sigma$ are disjoint. Hence the common perpendicular to $X_{2}$ and $X_{3}$ is a line $Y \subset \Sigma$. For $i=2,3$, the line $Y$ meets the line $X_{i} \subset \Pi_{i}$ perpendicularly, and $Y$ is contained in the plane $\Sigma$ which is perpendicular to $\Pi_{i}$; hence $Y$ is itself perpendicular to $\Pi_{i}$. It follows that $Y=\lambda_{23}$. Thus the plane $\Sigma$ contains $\lambda_{12}, \lambda_{13}$ and $\lambda_{23}$.

Lemma 2.4. Let $N$ be a compact hyperbolic 3-manifold with totally geodesic boundary, and suppose $\Pi_{1}, \Pi_{2}$ and $\Pi_{3}$ are distinct components of $\partial \tilde{N}$. Let $\Pi$ be the plane containing the short cuts $\lambda_{12}, \lambda_{23}$ and $\lambda_{13}$, which exists by Lemma 2.3. Let $C$ be the right-angled hexagon in $\Pi$ with edges $\lambda_{i j}$ and the geodesic arcs in the $\Pi_{i}$ joining their endpoints. Then $C \subset \tilde{N}$, and $C \cap \partial \widetilde{N}=$ $\cup_{i}\left(C \cap \Pi_{i}\right)$.

Proof. $\Pi \cap \widetilde{N}$ is a convex subset of $\Pi$ bounded by the family of disjoint geodesics $\Pi \cap \partial \widetilde{N}$, which includes $\Pi \cap \Pi_{i}, i \in\{1,2,3\}$. If $\gamma$ is another component of $\Pi \cap \partial \tilde{N}$, then by definition $\Pi \cap \Pi_{1}, \Pi \cap \Pi_{2}$ and $\Pi \cap \Pi_{3}$ are all contained in the component of $\Pi-\gamma$ intersecting $\widetilde{N}$. Thus there is a single component of $\partial_{\infty} \Pi-\left(\cup_{i=1}^{3} \partial_{\infty} \Pi_{i}\right)$ containing both endpoints of $\gamma$. Since $\gamma$ is a component of $\Pi \cap \partial \widetilde{N}$, its endpoints are between those of two different geodesics $\Pi \cap \Pi_{i}$, say $\Pi \cap \Pi_{1}$ and $\Pi \cap \Pi_{2}$. Then since the geodesic containing $\lambda_{12}$ intersects $\Pi_{1}$ and $\Pi_{2}$ perpendicularly, it is disjoint from $\gamma$ and contained in the component of $\Pi-\gamma$ intersecting $\tilde{N}$. The remainder of $C$ is on the other side of $\lambda_{12}$ from $\gamma$. Since $\gamma$ was arbitrary, the lemma follows.

Definition 2.5. Let $N$ be a compact hyperbolic 3-manifold with totally geodesic boundary, and let $C$ be a right-angled hexagon supplied by Lemma 
2.4. We call the edges of $C$ which are short cuts internal, and the remaining edges external. If the internal edges project to $\lambda_{i}, \lambda_{j}$ and $\lambda_{k}$, we call $C$ an $(i, j, k)$ hexagon.

This terminology matches that defined in [13] in the context of horospheres and cusped hyperbolic manifolds. As we will see below, $(i, j, k)$ hexagons were used extensively in the analysis of [17], although not by name.

The isometry class of a right-angled hexagon is determined by the lengths of three of its pairwise nonadjacent sides. If $\left\{\ell, \ell^{\prime}, \ell^{\prime \prime}\right\}$ is a collection of such lengths, and $d$ is the length of a side abutting those with lengths $\ell$ and $\ell^{\prime}$, the right-angled hexagon rule (cf., e.g., [19, Theorem 3.5.13]) describes $d$ in terms of the other lengths

$$
\cosh d=\frac{\cosh \ell \cosh \ell^{\prime}+\cosh \ell^{\prime \prime}}{\sinh \ell \sinh \ell^{\prime}}
$$

A prototypical application of the right-angled hexagon rule is the following initial lemma, proved in [17] during the proof of Lemma 3.2.

Lemma 2.6 (Kojima-Miyamoto). Suppose $N$ is a compact hyperbolic 3-manifold with connected totally geodesic boundary, and let $R$ be the function of $\ell_{1}$ defined by the following formula:

$$
\cosh R=\sqrt{1+\frac{1}{2 \cosh \ell_{1}-2}} .
$$

Then $d_{11} \geq 2 R$.

Proof. Let $\lambda$ and $\lambda^{\prime}$ be short cuts in $\tilde{N}$ with length $\ell_{1}$ whose feet are at distance $d_{11}$ on some boundary component. The short cut $\lambda^{\prime \prime}$ joining the boundary components containing the other feet of $\lambda$ and $\lambda^{\prime}$ has length $\ell_{k}$ for some $k \geq 1$. Applying the right-angled hexagon rule to the $(1,1, k)$ hexagon containing $\lambda, \lambda^{\prime}$ and $\lambda^{\prime \prime}$ yields the following inequality:

$$
\cosh d_{11}=\frac{\cosh ^{2} \ell_{1}+\cosh \ell_{k}}{\sinh ^{2} \ell_{1}} \geq \frac{\cosh ^{2} \ell_{1}+\cosh \ell_{1}}{\sinh ^{2} \ell_{1}}=1+\frac{1}{\cosh \ell_{1}-1} .
$$

The lemma now follows upon applying the "half-angle formula" for hyperbolic cosine, $\cosh R=\sqrt{(\cosh (2 R)+1) / 2}$.

Note that $R$ is decreasing as a function of $\ell_{1}$. Hence using Lemma 2.6, an upper bound on $d_{11}$ implies a lower bound on $\ell_{1}$. An upper bound for $d_{11}$ follows from area considerations. 
Lemma 2.7 ([17, Corollary 3.5]). Let $N$ be a compact hyperbolic 3-manifold with $\partial N$ connected, totally geodesic and of genus 2 . Then the following bounds hold for $d_{11}$ and $\ell_{1}$ :

$$
\cosh d_{11} \leq 3+2 \sqrt{3}, \quad \cosh \ell_{1} \geq \frac{3+\sqrt{3}}{4} .
$$

Proof. By Lemma 2.6, there is a disk of radius $R$ embedded on $\partial N$ around each endpoint of $\lambda_{1}$, and these two disks do not overlap. They lift to a radius $R$ disk packing on a component $\Pi$ of $\partial \widetilde{N}$, invariant under the action of $\pi_{1} \partial N$. Boröczky's Theorem [4] gives an upper bound $d(R)$ on the local density of a radius $R$ disk packing of $\mathbb{H}^{2}$. Since the packing in question is invariant under the action by covering transformations for the compact surface $\partial N$, $d(R)$ bounds the global density of the packing there, yielding the following inequality:

$$
\frac{4 \pi(\cosh R-1)}{4 \pi} \leq d(R)
$$

The numerator on the left-hand side of the inequality above is twice the area of a hyperbolic disk of radius $R$, and the denominator is the area of $\partial N$. (This follows from the Gauss-Bonnet theorem and the fact that $\partial N$ has genus 2.)

Let $\alpha$ be the angle at a vertex of a hyperbolic equilateral triangle $T(R)$ with side length $2 R . T(R)$ has area $\pi-3 \alpha$, and the intersection with $T(R)$ of disks of radius $R$ centered at its vertices occupies a total area of $3\left(\frac{\alpha}{2 \pi}\right) 2 \pi$ $(\cosh R-1)$. Boröczky's bound $d(R)$ is defined as the ratio of these areas; thus after simplifying the inequality above we obtain the one below:

$$
\cosh R-1 \leq \frac{3 \alpha(\cosh R-1)}{\pi-3 \alpha} .
$$

Solving for $\alpha$ yields $\alpha \geq \pi / 6$. The hyperbolic law of cosines describes the relationship between $\alpha$ and the side length of $T(R)$ :

$$
\cos \alpha=\frac{\cosh ^{2}(2 R)-\cosh (2 R)}{\sinh ^{2}(2 R)}=\frac{\cosh (2 R)}{\cosh (2 R)+1}=\frac{2 \cosh ^{2} R-1}{2 \cosh ^{2} R} .
$$

Using the fact that $\cos \alpha \leq \sqrt{3} / 2$ and solving for $\cosh R$ yields $\cosh R \leq$ $(1+\sqrt{3}) / \sqrt{2}$. The inequality for $\cosh d_{11}$ follows using the "hyperbolic double angle formula," and the inequality for $\cosh \ell_{1}$ follows upon solving the formula of Lemma 2.6.

The following lemma combines Lemmas 4.2 and 4.3 of [17] with the discussion below them. 
Lemma 2.8 (Kojima-Miyamoto). Let $N$ be a compact hyperbolic 3-manifold with $\partial N$ totally geodesic connected, and of genus 2. Define quantities $R^{\prime}, E$ and $F$, depending on $\ell_{1}$, by the following equations:

$$
\begin{aligned}
\cosh R^{\prime} & =3-\cosh R, \\
\cosh E & =\frac{2}{\cosh ^{2}\left(R+R^{\prime}\right) \tanh ^{2} \ell_{1}-1}+1, \\
\cosh F & =\sqrt{\frac{\cosh \ell_{1}+1}{\cosh 2 R^{\prime}-1}+1} .
\end{aligned}
$$

Then $\ell_{2} \geq \max \left\{\ell_{1}, \min \{E, F\}\right\}$.

Proof. The right-angled hexagon rule can be used to obtain lower bounds on $\ell_{2}$ depending on values for $\ell_{1}$ and $d_{12}$ or $d_{22}$, respectively:

$$
\begin{aligned}
\cosh \ell_{2} & \geq \frac{2}{\cosh ^{2} d_{12} \tanh ^{2} \ell_{1}-1}+1, \\
\cosh \ell_{2} & \geq \sqrt{\frac{\cosh \ell_{1}+1}{\cosh d_{22}-1}+1 .}
\end{aligned}
$$

This is recorded in Lemma 4.2 of [17]. Therefore an upper bound for $d_{12}$ or $d_{22}$ gives a lower bound for $\ell_{2}$ in terms of $\ell_{1}$.

Recall from above that an upper bound on $\ell_{1}$ gives a lower bound of $2 R\left(\ell_{1}\right)$, on $d_{11}$, the shortest distance between feet of two different shortest short cuts on (any) component of $\partial \widetilde{N}$. Hence disks $U$ and $U^{\prime}$ of radius $R$ in $\partial N$, each centered at a foot of the shortest return path, are embedded and nonoverlapping. Area considerations imply that $R^{\prime}$ is an upper bound for the radii of two equal-size nonoverlapping disks in $\partial N-\operatorname{int}\left(U \cup U^{\prime}\right)[17$, Lemma 4.3]. It follows that at least one of $d_{12} \leq R+R^{\prime}$ or $d_{22} \leq 2 R^{\prime}$ holds, since otherwise there would be disks of radius $R^{\prime}$ embedded around the feet of the second-shortest return path without overlapping $U$ and $U^{\prime}$. Inequalities (2.8.4) and (2.8.5) thus imply that $\ell_{2} \geq \min \{E, F\}$. Note that by definition $\ell_{2} \geq \ell_{1}$, which gives the lemma.

The following lemma contains a new observation improving on the bound of Lemma 2.8 for values of $\ell_{1}$ with hyperbolic cosine near 1.4.

Lemma 2.9. Let $N$ be a compact hyperbolic 3-manifold with $\partial N$ totally geodesic, connected and of genus 2 . Let $R^{\prime \prime}$ be determined by the following 
equation:

$$
\cosh R^{\prime \prime}=\frac{1}{\sqrt{2(1-\cos (2 \pi / 9))}}=1.4619 \ldots
$$

Define quantities $L$ and $M$ depending on $\ell_{1}$ by

$$
\begin{aligned}
& \cosh L=\frac{2}{\cosh ^{2}\left(2 R^{\prime \prime}\right) \tanh ^{2} \ell_{1}-1}+1, \\
& \cosh M=\sqrt{\frac{\cosh \ell_{1}+1}{\cosh \left(2 R^{\prime \prime}\right)-1}+1 .}
\end{aligned}
$$

For any value of $\ell_{1}$ with

$$
\cosh \ell_{1} \leq \frac{\cos (2 \pi / 9)}{2 \cos (2 \pi / 9)-1}=1.439 \ldots,
$$

$\ell_{2}$ is bounded below by $\max \left\{\ell_{1}, \min \{E, F\}, \min \{L, M\}\right\}$.

Proof. Applying Boröczky's theorem as in Lemma 2.7, but this time to four disks of equal radius packed on $\partial N$, we find that the radius is bounded above by the quantity $R^{\prime \prime}$ specified by the formula above. For $\ell_{1}$ satisfying the bound of (2.9.4), inequality (2.6.2) implies that $d_{11}$ is at least $2 R^{\prime \prime}$. If each of $d_{12}$ and $d_{22}$ were also larger than $2 R^{\prime \prime}$, then disks of radius $R^{\prime \prime}$ around the feet of both the shortest and second-shortest return paths would be embedded and nonoverlapping, a contradiction. Thus $\min \left\{d_{12}, d_{22}\right\} \leq 2 R^{\prime \prime}$. Plugging into inequalities (2.8.4) and (2.8.5) gives the result.

The quantities $L$ and $M$ defined above offer a better lower bound on $\ell_{2}$ than $E$ and $F$, for values of $\ell_{1}$ with $1.367 \leq \cosh \ell_{1} \leq 1.439$. This is because $E$ and $F$ use the quantity $R^{\prime}$ of equation (2.8.1), and by definition twice the area of a hyperbolic disk of radius $R^{\prime}$ plus twice the area of a hyperbolic disk of radius $R$ is $4 \pi$. However, it is impossible to entirely cover a compact surface of genus 2 with 4 nonoverlapping embedded disks. Boröczky's theorem bounds the proportion of the area which can be covered by four disks of equal radius, and this supplies $R^{\prime \prime}$. This is less than $R^{\prime}$ for $R$ determined below:

$$
\cosh R \leq 3-\frac{1}{\sqrt{2(1-\cos (2 \pi / 9))}} .
$$

Solving Equation (2.6.1) for $\cosh \ell_{1}$, we find that this occurs when $\cosh \ell_{1} \geq$ $1.366 \ldots$ It is at this point that the bound above for $d_{22}$ given by $\cosh (K)$ 
is better than the bound above given by $2 R^{\prime}$. The bound on $d_{12}$ given by $\cosh (K)$ becomes better than that given by $R+R^{\prime}$ somewhat earlier, at least for values of $\ell_{1}$ with $\cosh \ell_{1} \geq 1.25$.

\section{Volume with a long return path}

By definition, in a hyperbolic manifold $N$ with totally geodesic boundary, $\ell_{1} / 2$ is the height of a maximal embedded collar of $\partial N$. The volume of such a collar bounds the volume of $N$ below, but leaves a lot out. The volume bounds of [17] are obtained by taking a larger collar of $\partial N$ and using separate means to understand the region where it overlaps itself.

Definition 3.1. The muffin of height $\ell$, here denoted $\mathrm{Muf}_{\ell}$, is the hyperbolic solid obtained by rotating a hyperbolic pentagon with base of length $\ell$, opposite angle $2 \pi / 3$, and all other angles $\pi / 2$, about its base (see [17, Figure 3.1]).

It is a standard fact of hyperbolic trigonometry (see [19, Theorem 3.5.14]) that positive real numbers $a, b$ and $c$ determine a right-angled hexagon in $\mathbb{H}^{2}$, unique up to isometry, with alternate sides of lengths $a, b$ and $c$. For $\ell>0$, the hexagon specified by $a=b=c=\ell$ has an orientation preserving symmetry group of order three which cyclically permutes the sides of length $\ell$. The hyperbolic pentagon mentioned in the definition above is a fundamental domain for this symmetry group.

For a compact hyperbolic 3-manifold $N$, a copy of $\mathrm{Muf}_{\ell_{1}}$ is embedded in $\widetilde{N}$ around each short cut with length $\ell_{1}$. Lemma 3.2 of [17] asserts that Muf $_{\ell_{1}}$ is embedded in $N$ by the universal covering. Let $A$ be the length of a side joining a vertex with angle $\pi / 2$ to the vertex with angle $2 \pi / 3$ of the pentagon rotated to construct the muffin. In terms of $\ell_{1}, A$ is given by the formula below:

$$
\cosh A=\sqrt{\frac{2}{3}\left(\cosh \ell_{1}+1\right)} .
$$

A collar of $\partial N$ with height less than both $A$ and $\ell_{2} / 2$ has its region of selfoverlap entirely contained in $\mathrm{Muf}_{\ell_{1}}$. This yields the fundamental volume inequality of [17], stated there in the proof of Proposition 4.1, which we formulate in the following lemma.

Lemma 3.2 (Kojima-Miyamoto). Let $N$ be a compact hyperbolic 3manifold with totally geodesic boundary, and let $H=\min \left\{A, \ell_{2} / 2\right\}$. With 


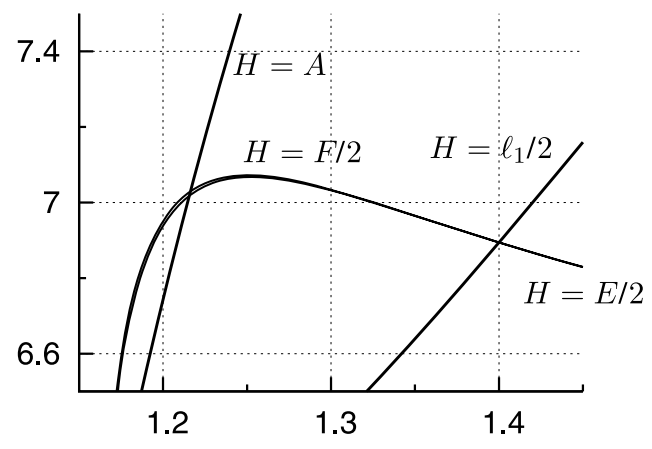

Figure 3.1: The volume estimates of Kojima-Miyamoto.

Muf $_{\ell_{1}}$ as defined above and $R$ as in Lemma 2.6, we have the following bound:

$$
\operatorname{vol}(N) \geq \operatorname{vol}\left(\operatorname{Muf}_{\ell_{1}}\right)+\pi(2-\cosh R)(2 H+\sinh (2 H))
$$

Proof. The intersection of $\mathrm{Muf}_{\ell_{1}}$ with $\partial N$ is the union of disks $U$ and $U^{\prime}$ of radius $R$, the quantity defined in Lemma 2.6. This is because the pentagon rotated to construct the muffin is a fundamental domain for the orientationpreserving symmetry group of a $(1,1,1)$ hexagon, thus its sides adjacent to the base each have length $R$ (again see [17, Figure 3.1]). It follows from Lemma 2.6 that $U$ and $U^{\prime}$ are embedded in $\partial N$ without overlapping.

The area of $\partial N-\left(U \cup U^{\prime}\right)$ is $4 \pi-4 \pi(\cosh R-1)$. A collar of $\partial N-$ $\left(U \cup U^{\prime}\right.$ ) of height $H$ is embedded in $N$ without overlapping Muf $_{\ell_{1}}$, and the bound of the lemma is obtained by adding their volumes. This uses the following well-known formula for the volume $V$ of a collar of height $H$ in $\mathbb{H}^{3}$ of a set in a plane with area $A: V=A(2 H+\sinh 2 H) / 4$.

A formula for the volume of $\mathrm{Muf}_{\ell_{1}}$ is recorded in [17, Lemma 3.3]. The output of inequality (3.2.1) is recorded as a function of $x=\cosh \ell_{1}$ in $[17$, Graph 4.1], which we have reproduced above in figure 3.1. Lower bounds for $H$ are determined at various points on the graph by $A$, the quantities $E$ and $F$ of Lemma 2.8, and $\ell_{1}$ itself. The point on Graph 4.1 of [17] above $\cosh \ell_{1}=1.215$ is just to the left of the intersection of the curves labeled $H=A$ and $H=F / 2$. A computation gives a volume bound of $7.007 \ldots$ here. To the right of $\cosh \ell_{1}=1.215$, inspection reveals a single local minimum at about $\cosh \ell_{1}=1.4$. Numerical experimentation indicates that the volume bound at this minimum is just larger than 6.89. In this section, our main task is to prove this rigorously. 
Proposition 3.7. Let $N$ be a hyperbolic 3-manifold with $\partial N$ connected, totally geodesic and of genus 2 , satisfying $\cosh \ell_{1} \geq 1.215$. Then $N$ has volume greater than 6.89 .

Remark 3.3. Although Proposition 3.7 probably follows solely from results of [17], our proof uses Lemma 2.9. In fact, numerical experimentation indicates that the volume bound above could be improved to about 6.94 with this result. Since it is easier and suffices for applications, we prove only the bound of 6.89 here.

The lemma below proves useful for several results in this section.

Lemma 3.4. For $\frac{3+\sqrt{3}}{4} \leq \cosh \ell_{1} \leq 1.4$, the quantity $E$ of $(2.8 .2)$ is decreasing in $\ell_{1}$.

Proof. Formula (2.8.1) defines $R^{\prime}$ in terms of $R$ by

$$
R^{\prime}=\cosh ^{-1}(3-\cosh R)=\log \left[(3-\cosh R)+\sqrt{(3-\cosh R)^{2}-1}\right] .
$$

Taking a derivative with respect to $R$, one finds that $R+R^{\prime}$ increases with $R$ for $1<\cosh R<3 / 2$, reaching a maximum at $\cosh R=3 / 2$, and decreases when $\cosh R>3 / 2$. This implies that $\cosh \left(R+R^{\prime}\right)$ is an increasing function of $\ell_{1}$ on the interval $\frac{3+\sqrt{3}}{4} \leq \cosh \ell_{1} \leq 1.4$, since values of $\ell_{1}$ in this interval give $R$-values between $\frac{3}{2}$ and $\frac{1+\sqrt{3}}{\sqrt{2}}$, and $R$ is a decreasing function of $\ell_{1}$. Since the hyperbolic tangent is an increasing function, the lemma follows from the definition of $E$ in Lemma 2.8.

In the proof of Proposition 3.7, we divide the interval $1.215 \leq \cosh \ell_{1}<$ $\infty$ into subintervals:

$$
[1.215, \infty)=[1.215,1.367] \cup[1.367,1.439] \cup[1.439, \infty) .
$$

We address each subinterval separately. The first is below.

Lemma 3.5. A compact hyperbolic 3-manifold $N$ with geodesic boundary satisfying $1.215 \leq \cosh \ell_{1} \leq 1.367$ has volume greater than 6.89 .

Proof. The strategy of proof is to break the interval in question into subintervals, bound the constituent quantities in formula (3.2.1) on each subinterval, and from this obtain a coarse lower bound for the right-hand side 
Table 1: Data for the proof of Lemma 3.5.

\begin{tabular}{lcccc}
\hline $\cosh \ell_{1}$ & Muffin volume & $\operatorname{area}\left(\partial N-\left(U \cup U^{\prime}\right)\right)$ & $H$ & Volume \\
\hline$[1.215,1.220]$ & 5.304 & 2.216 & $0.629(E)$ & 6.899 \\
{$[1.220,1.226]$} & 5.236 & 2.399 & $0.611(E)$ & 6.899 \\
{$[1.226,1.233]$} & 5.159 & 2.609 & $0.592(E)$ & 6.900 \\
{$[1.233,1.241]$} & 5.076 & 2.844 & $0.574(E)$ & 6.901 \\
{$[1.241,1.250]$} & 4.988 & 3.097 & $0.556(E)$ & 6.901 \\
{$[1.250,1.260]$} & 4.895 & 3.367 & $0.539(F)$ & 6.898 \\
{$[1.260,1.270]$} & 4.808 & 3.648 & $0.524(F)$ & 6.908 \\
{$[1.270,1.281]$} & 4.717 & 3.911 & $0.510(F)$ & 6.898 \\
{$[1.281,1.292]$} & 4.632 & 4.182 & $0.498(F)$ & 6.900 \\
{$[1.292,1.303]$} & 4.551 & 4.436 & $0.488(F)$ & 6.898 \\
{$[1.303,1.314]$} & 4.475 & 4.675 & $0.479(F)$ & 6.894 \\
{$[1.314,1.324]$} & 4.409 & 4.899 & $0.471(F)$ & 6.899 \\
{$[1.324,1.334]$} & 4.346 & 5.092 & $0.464(F)$ & 6.891 \\
{$[1.334,1.343]$} & 4.292 & 5.275 & $0.459(F)$ & 6.893 \\
{$[1.343,1.351]$} & 4.245 & 5.432 & $0.454(F)$ & 6.894 \\
{$[1.351,1.358]$} & 4.206 & 5.565 & $0.451(F)$ & 6.895 \\
{$[1.358,1.364]$} & 4.173 & 5.678 & $0.448(F)$ & 6.896 \\
{$[1.364,1.367]$} & 4.157 & 5.772 & $0.447(F)$ & 6.917 \\
\hline
\end{tabular}

of the inequality. Table 1 records this computation. We explain its entries below.

The leftmost column specifies the subinterval of values of $\cosh \ell_{1}$. The second column records a lower bound on this interval for the volume of the muffin. This is attained at the right endpoint, according to $[17$, Lemma 3.3]. The third column records a lower bound for the area on $\partial N$ of the complement of the base of the muffin. This is attained at the left endpoint of the subinterval, since $R$ is decreasing in $\ell_{1}$. The fourth column records a lower bound for $H$. This is obtained by computing minima for each of $A$, $E / 2$ and $F / 2$ on the subinterval and taking the minimum (in each case this lower bound is greater than $\left.\ell_{1} / 2\right)$.

From its definition (3.1.1) one easily finds that $A$ is increasing in $\ell_{1}$, hence a minimum for $A$ is obtained at the left endpoint of each subinterval. By Lemma 3.4 above, $E$ is decreasing in $\ell_{1}$ on each subinterval in question, so its minimum is obtained at the right endpoint. The monotonicity of $F$ is not apparent from its definition, so to find a lower bound on each subinterval, we plug the value of $\cosh \ell_{1}$ at the left endpoint and the value of $\cosh 2 R^{\prime}$ 
at the right endpoint to formula (2.8.3). In addition to the resulting lower bound, we record which of $A, E$ and $F$ supplies it in the fourth column of table 1.

The final column assembles these bounds to give a volume bound. In each column after the first, the decimal approximation has been truncated after three places.

Using the bounds of Lemma 2.9 for $\ell_{2}$ in inequality (3.2.1) yields the lemma below.

Lemma 3.6. A compact hyperbolic 3-manifold with geodesic boundary satisfying $1.367 \leq \cosh \ell_{1} \leq 1.439$ has volume at least 6.89 .

Proof. We assemble a table as in the proof of Lemma 3.5, using $L$ and $M$ to bound $H$ below, instead of $E$ and $F$. Since $L$ is decreasing in $\ell_{1}$, its minimum occurs at the right endpoint of each subinterval, whereas the minimum of $M$ occurs at the left endpoint. The results of the computation are recorded in table 2 .

Table 2: Data for the proof of Lemma 3.6.

\begin{tabular}{lcccc}
\hline $\cosh \ell_{1}$ & Muffin volume & $\operatorname{area}\left(\partial N-\left(U \cup U^{\prime}\right)\right)$ & $H$ & Volume \\
\hline$[1.367,1.377]$ & 4.105 & 5.818 & $0.447(M)$ & 6.892 \\
{$[1.377,1.392]$} & 4.031 & 5.966 & $0.448(M)$ & 6.894 \\
{$[1.392,1.416]$} & 3.920 & 6.176 & $0.449(M)$ & 6.893 \\
{$[1.416,1.439]$} & 3.823 & 6.485 & $0.451(M)$ & 6.959 \\
\hline
\end{tabular}

We now prove Proposition 3.7. Below we recall its statement.

Proposition 3.7. Let $N$ be a hyperbolic 3-manifold with $\partial N$ connected, totally geodesic and of genus 2 , satisfying $\cosh \ell_{1} \geq 1.215$. Then $N$ has volume greater than 6.89 .

Proof. Given Lemmas 3.5 and 3.6, we need only concern ourselves with hyperbolic 3 -manifolds $N$ with totally geodesic boundary satisfying $1.439 \leq$ $\cosh \ell_{1}$. The union of the muffin and a collar of height $H=\ell_{1} / 2$ has volume 
given by the following formula:

$$
\begin{aligned}
V\left(\ell_{1}\right)=\pi & {\left[\ell_{1}+2 \sinh \ell_{1}+\sqrt{\frac{2 \cosh \ell_{1}-1}{2 \cosh \ell_{1}-2}}\right.} \\
& \left.\times\left(\cosh ^{-1}\left(\frac{4 \cosh \ell_{1}+1}{3}\right)-\ell_{1}-\sinh \ell_{1}\right)\right] .
\end{aligned}
$$

When $\cosh \ell_{1}=1.439$, this yields a volume of $7.1 \ldots$ We claim that $V\left(\ell_{1}\right)$ is increasing with $\ell_{1}$ (as is suggested by Graph 4.1 in [17]). Once established, this will complete the proof.

It is clear that $\sqrt{\frac{2 \cosh \ell_{1}-1}{2 \cosh \ell_{1}-2}}=\cosh R$ (recall Equation (2.6.1)) is decreasing in $\ell_{1}$, asymptotically approaching 1 from above. The derivative of $\cosh ^{-1}$ $\left(\left(4 \cosh \ell_{1}+1\right) / 3\right)$ is $1 / \cosh R$, bounded above by one. Hence the quantity in parentheses above,

$$
Q=\cosh ^{-1}\left(\frac{4 \cosh \ell_{1}+1}{3}\right)-\ell_{1}-\sinh \ell_{1}
$$

is decreasing in $\ell_{1}$. Its value is negative when $\cosh \ell_{1}=1.439$, and so also on the entire interval in question. Taking the derivative of $V\left(\ell_{1}\right)$ yields the following.

$$
V^{\prime}\left(\ell_{1}\right)=\pi\left[1+2 \cosh \ell_{1}+\cosh R\left(\frac{1}{\cosh R}-1-\cosh \ell_{1}\right)+\frac{d}{d \ell_{1}}(\cosh R) Q\right] .
$$

Since $\frac{d}{d \ell_{1}}(\cosh R)$ and $Q$ are both negative, the above is the sum of a positive number with the product of $1+\cosh \ell_{1}$ and $2-\cosh R$. When $\cosh \ell_{1}=$ $1.439, \cosh R=1.46 \ldots<2$; since $\cosh R$ is decreasing as a function of $\ell_{1}$, the derivative of the volume formula is positive for $\cosh \ell_{1} \geq 1.439$.

Lemma 3.8. Let $N$ be a compact hyperbolic 3-manifold with $\partial N$ connected, totally geodesic and of genus 2 . If $\cosh \ell_{1} \leq 1.215$, then $\ell_{1}$ and $A$ are each less than $\ell_{2} / 2$.

Proof. Lemma 2.8 implies that $\ell_{2} \geq \min \{E, F\}$. The values of $\ell_{1}$ in question here have hyperbolic cosine between $\frac{3+\sqrt{3}}{4} \cong 1.186$ and 1.215. By Lemma 3.4, $E$ is monotone decreasing in $\ell_{1}$ on this interval; thus a lower bound, obtained by plugging in 1.215 , is $1.961 \ldots$ The quantity $F$ is not necessarily monotone in $\ell_{1}$, since both $\cosh \ell_{1}$ and $\cosh 2 R^{\prime}$ are increasing in $\ell_{1}$. However, a coarse lower bound may be found by substituting $\cosh ^{-1}(1.215)$ for $\ell_{1}$ in $\cosh \ell_{1}$ and $\cosh ^{-1}\left(\frac{3+\sqrt{3}}{4}\right)$ for $\ell_{1}$ in $\cosh 2 R^{\prime}$. The lower bound obtained in this way 
is $1.960 \ldots$ When $\cosh \ell_{1} \leq 1.215, \ell_{1} \leq .645$. Taking the inverse hyperbolic cosine of 1.960 , we find $\ell_{2} \geq 1.293$. The quantity $A$ is clearly increasing in $\ell_{1}$. When $\cosh \ell_{1}=1.215$, the value obtained is $.644 \ldots$ This establishes the lemma.

Proposition 3.9. Let $N$ be a hyperbolic 3-manifold with $\partial N$ connected, totally geodesic and of genus 2 , such that there is no $(1,1,1)$ hexagon in $\tilde{N}$. Then $\cosh \ell_{1} \geq 1.215$.

Proof. Suppose $N$ is a hyperbolic 3-manifold with totally geodesic boundary and no $(1,1,1)$ hexagons. According to Lemma 3.8, if $\ell_{1}$ is at most 1.215, then $\ell_{2}$ is at least twice $\ell_{1}$. Since $N$ has no $(1,1,1)$ hexagons, in the rightangled hexagon in $\widetilde{N}$ realizing $d_{11}$, the opposite side has length at least $\ell_{2}$; thus at least twice $\ell_{1}$. Using the right-angled hexagon rule as in inequality (2.6.2), we obtain the following inequality:

$$
\cosh d_{11} \geq \frac{\cosh ^{2} \ell_{1}+\cosh \left(2 \ell_{1}\right)}{\sinh ^{2} \ell_{1}}=\frac{3 \cosh ^{2} \ell_{1}-1}{\cosh ^{2} \ell_{1}-1}=3+\frac{2}{\sinh ^{2} \ell_{1}} .
$$

We recall from Lemma 2.7 that $\cosh d_{11} \leq 3+2 \sqrt{3}$. Putting this together with the inequality above implies

$$
\sinh ^{2} \ell_{1} \geq \frac{1}{\sqrt{3}}
$$

This gives $\cosh \ell_{1}>1.255$, contradicting the hypothesis that $\cosh \ell_{1} \leq 1.215$.

\section{Normal books of I-bundles}

4.1. The results of this section and the next are topological. We work in the PL category in these sections, and follow the conventions of [6]. In particular, we say that a subset $Y$ of a space $X$ is $\pi_{1}$-injective if for all path components $A$ of $X$ and $B$ of $Y$ such that $B \subset A$, the inclusion homomorphism $\pi_{1}(B) \rightarrow$ $\pi_{1}(A)$ is injective. We denote the Euler characteristic of a finite polyhedron $X$ by $\chi(X)$, and we write $\bar{\chi}(X)=-\chi(X)$.

This section collects topological results to be used in the sequel. After some preliminary results and definitions, we define a normal book of Ibundles in 4.6 below, and follow with the main results: Proposition 4.7 gives a criterion (equivalent to having empty "kishkes," or "guts") for recognizing a simple 3-manifold as the underlying topological space of a normal 
book of $I$-bundles, and Lemma 4.9 identifies a property satisfied by certain submanifolds of a normal book of $I$-bundles.

Proposition 4.2. Let $X$ be a compact, orientable 3-manifold, and let $S$ and $T$ be components of $\partial X$. Suppose that for some field $F$ the inclusion homomorphism $H_{1}(T ; F) \rightarrow H_{1}(X ; F)$ is surjective. Then $S$ is $\pi_{1}$-injective in $X$.

Proof. Assume that $S$ is not $\pi_{1}$-injective. Then there is a properly embedded disk $D \subset X$ such that $\partial D$ is a nontrivial simple closed curve in $S$. Let $N$ be a regular neighborhood of $D$ in $X$, let $A$ denote the component of $\overline{X-N}$ containing $T$, let $\star$ be a base point in $T$, and let $G \leq \pi_{1}(X, \star)$ denote the image of $\pi_{1}(T, \star)$ under the inclusion homomorphism. Then $\pi_{1}(X, \star)$ is the free product of $G$ with another subgroup $K$, where $K \cong \mathbb{Z}$ if $D$ does not separate $X$, and $K \cong \pi_{1}(\overline{X-A})$ if $D$ does separate $X$. In the latter case, $\partial D$ is a separating, nontrivial simple closed curve in $S$, and hence $\overline{X-A}$ has a boundary component of strictly positive genus. Thus in either case we have $H_{1}(K ; F) \neq 0$. Hence the inclusion homomorphism $H_{1}(A ; F) \rightarrow H_{1}(X ; F)$ is not surjective. Since $S \subset A$, this contradicts the hypothesis.

Lemma 4.3. Let $\tau: F \rightarrow F$ be a free involution of a compact orientable surface $F$, and let $C \subset F$ be a simple closed curve. Suppose that $\tau(C)$ is isotopic to a curve which is disjoint from $C$. Then $C$ is isotopic to a curve $C_{1}$ such that either (i) $\tau\left(C_{1}\right) \cap C_{1}=\emptyset$ or (ii) $\tau\left(C_{1}\right)=C_{1}$. Furthermore, if $\tau$ reverses orientation we may always choose $C_{1}$ so that (i) holds.

Proof. We fix a metric with convex boundary on $F /\langle\tau\rangle$. Then $F$ inherits a metric such that $\tau: F \rightarrow F$ is an isometry. Since $C$ is a homotopically nontrivial simple closed curve in $F$, it is homotopic to a curve $C_{1}$ with shortest length in its homotopy class, which is a simple closed geodesic by freedman et al. [11, Theorem 2.1]. Let $C^{\prime}$ be homotopic to $\tau(C)$ and disjoint from $C$. Then since the shortest closed geodesics $C_{1}$ and $\tau\left(C_{1}\right)$ are, respectively, homotopic to the disjoint curves $C$ and $C^{\prime}$, it follows from [11, Corollary 3.4] that they either are disjoint or coincide. (The results we have quoted from [11] are stated there for the case of a closed surface, but it is pointed out in the first paragraph of $[11, \S 4]$ that they hold in the case of a compact surface with convex boundary.)

It follows from [10, Theorem 2.1] that $C$ is isotopic to $C_{1}$. This proves the first assertion.

To prove the second assertion, suppose that $\tau$ reverses orientation and that $C$ is isotopic to a curve $C_{1}$ such that (ii) holds. Let $A$ be an invariant 
annular neighborhood of $C_{1}$. Since $\tau$ is a free involution it must preserve an orientation of the invariant curve $C$; since it reverses an orientation of $F$, it must therefore interchange the components of $\partial A$. Hence (i) holds if $C_{1}$ is replaced by one of the components of $\partial A$.

If a 3-manifold $X$ has the structure of an $I$-bundle over a surface $T$ and $p: X \rightarrow T$ is the bundle projection, we will call $\partial_{v} X \doteq p^{-1}(\partial T)$ the vertical boundary of $X$ and $\partial_{h} X \doteq \overline{\partial X-\partial_{v} X}$ the horizontal boundary of $X$. Note that $\partial_{v} X$ inherits the structure of an $I$-bundle over $\partial T$, and $\partial_{h} X$ the structure of a $\partial I$-bundle over $T$, from the original $I$-bundle structure on $X$. We call an annulus $A \subset X$ vertical if $A=p^{-1}(p(A))$.

Let $M$ an orientable, irreducible 3-manifold $M$, and let $F$ be a $\pi_{1^{-}}$ injective 2-dimensional submanifold of $\partial M$. By an essential annulus or torus in $M$ relative to $F$ we mean a properly embedded annulus or torus in $M$ which is $\pi_{1}$-injective, has its boundary contained in $F$, and not parallel to a subsurface of $F$.

In the case where $M$ is boundary-irreducible, we shall define an essential annulus or torus in $M$ to be an essential annulus or torus in $M$ relative to $\partial M$.

Proposition 4.4. Let $X$ be an I-bundle over a compact surface, and suppose that $A$ is an essential annulus in $X$ relative to $\partial_{h} X$. Then $A$ is isotopic, by an ambient isotopy of $X$ which is constant on $\partial_{v} X$, to a vertical annulus in $X$.

Proof. Let $j: A \rightarrow X$ denote the inclusion. If $j$ is homotopic to a map of $A$ into $\partial_{h} X$ then by Waldhausen [21, Lemma 5.3], $A$ is boundary parallel, contradicting essentiality relative to $\partial_{h} X$. Hence $j$ is not homotopic to a map of $A$ into $\partial_{h} X$.

It follows that if $X$ is a trivial $I$-bundle then $A$ has one boundary component on each component of $\partial_{h} X$. Lemma 3.4 of [21] then asserts that $A$ is ambiently isotopic in $X$ to a vertical annulus, by an isotopy fixing $\partial_{v} X$ and one component of $\partial_{h} X$. This gives the conclusion in the case where $X$ is a trivial $I$-bundle.

We turn to the case where $X$ is a twisted $I$-bundle. Then for some compact orientable surface $F$ and some orientation-reversing free involution $\tau: F \rightarrow F$ we may write $X=(F \times I) / \hat{\tau}$, where $\hat{\tau}: F \times I \rightarrow F \times I$ is defined by $\hat{\tau}(x, t)=(\tau(x), 1-t)$. The quotient map $p: F \times I \rightarrow X$ is a two-sheeted covering map, and $p$ maps $F \times\{i\}$ homeomorphically onto $\partial_{h} X$ for $i=0,1$. Hence $j: A \rightarrow X$ may be lifted to an embedding $\widetilde{j}: A \rightarrow F \times I$. Since $j$ is not homotopic to a map of $A$ into $\partial_{h} X$, the annulus $\widetilde{j}(A)$ is essential. By 
the case of the proposition already proved, $\widetilde{j}$ is ambiently isotopic in $F \times I$ to an embedding $\widetilde{j^{\prime}}$ such that $\widetilde{j^{\prime}}(A)$ is vertical.

For $i=0,1$ let $C_{i}$ denote the component of $\partial A$ with $\tilde{j}^{\prime}\left(C_{i}\right) \subset F \times\{i\}$. Define a simple closed curve $C \subset F$ by $C \times\{0\}=\widetilde{j}^{\prime}\left(C_{0}\right)$. Then $C \times\{0\}$ is isotopic to $\tilde{j}\left(C_{0}\right)$. Since $\widetilde{j}^{\prime}(A)$ is vertical, $\tilde{j}^{\prime}\left(C_{1}\right)=C \times\{1\}$; hence by definition we have $\tau(C) \times\{0\}=\hat{\tau}\left(\widetilde{j^{\prime}}\left(C_{1}\right)\right)$, and so $\tau(C) \times\{0\}$ is isotopic to $\hat{\tau}\left(\widetilde{j}\left(C_{1}\right)\right)$. But since $j$ is an embedding, $\widetilde{j}\left(C_{0}\right)$ and $\hat{\tau}\left(\widetilde{j}\left(C_{1}\right)\right.$ are disjoint curves in $F \times\{0\}$. Thus $C$ and $\tau(C)$ are isotopic in $F$ to disjoint curves, and it follows from Lemma 4.3 that $C$ is isotopic in $F$ to a curve $C_{1}$ such that $\tau\left(C_{1}\right) \cap C_{1}=\emptyset$. This implies that $\widetilde{j}^{\prime}$ is ambiently isotopic to an embedding $\widetilde{j_{1}^{\prime}}$ such that $\widetilde{j}_{1}^{\prime}(A)$ is a vertical annulus and $\tau\left(\widetilde{j_{1}^{\prime}}(A)\right) \cap \widetilde{j_{1}^{\prime}}(A)=\emptyset$.

Hence $p \circ{\widetilde{j_{1}^{\prime}}}_{1}^{\prime} A \rightarrow X$ is an embedding, and $A_{1} \doteq p \circ \widetilde{j}_{1}^{\prime}(A)$ is a vertical annulus in $X$. Since $\widetilde{j}_{1}^{\prime}$ is ambiently isotopic in $F \times I$ to $\widetilde{j}$, the homeomorphism $p \circ \widetilde{j}_{1}^{\prime}: A \rightarrow A_{1}$ is homotopic to $j$ by a boundary-preserving homotopy in $X$. It then follows from [21, Corollary 5.5] that $A$ is isotopic to $A_{1}$ by an ambient isotopy fixing $\partial_{v} X$.

4.5. Let $W$ be a compact, orientable, irreducible and boundary-irreducible 3 -manifold with $\partial W \neq \emptyset$. We recall the definition of the characteristic submanifold $\Sigma$ of $W$ relative to $\partial W$. Up to ambient isotopy, $\Sigma$ is the unique compact submanifold of $W$ with the following properties:

(1) Every component of $\Sigma$ is either an $I$-bundle $P$ over a surface such that $P \cap \partial W=\partial_{h} P$, or a Seifert fibered space $S$ such that $S \cap \partial W$ is a saturated 2-manifold in $\partial S$.

(2) Every component of the frontier of $\Sigma$ is an essential annulus or torus in $W$.

(3) No component of $\Sigma$ is ambiently isotopic in $W$ to a submanifold of another component of $\Sigma$.

(4) If $\Sigma_{1}$ is a compact submanifold of $W$ such that (1) and (2) hold with $\Sigma_{1}$ in place of $\Sigma$, then $\Sigma_{1}$ is ambiently isotopic in $W$ to a submanifold of $\Sigma$.

Recall that a submanifold of a Seifert fibered space $M$ is saturated if it is a union of fibers. For further details on the characteristic submanifold, see $[14,15]$.

It follows from property (2) of $W$ that $\Sigma$ is $\pi_{1}$-injective. Hence if $W$ is simple, the fundamental group of a component of $\Sigma$ cannot have a rank- 2 free abelian subgroup. It follows that if $W$ is simple then every Seifert-fibered 
component of $\Sigma$ is a solid torus. In particular, all the components of the frontier of $\Sigma$ are essential annuli in this case.

The rest of this section is concerned with books of $I$-bundles. We shall follow the conventions of [6], and we refer the reader to $[6,5.1]$ for the definition of a book of $I$-bundles. As in [6] we shall denote the union of the pages of a book of $I$-bundles by $\mathcal{P}_{\mathcal{W}}$ and the union of its bindings by $\mathcal{B}_{\mathcal{W}}$, and we shall set $|\mathcal{W}|=\mathcal{P}_{\mathcal{W}} \cup \mathcal{B}_{\mathcal{W}}$ and $\mathcal{A}_{\mathcal{W}}=\mathcal{P}_{\mathcal{W}} \cap \mathcal{B}_{\mathcal{W}}$.

Definition 4.6. Let $\mathcal{W}$ be a book of $I$-bundles, and set $W=|\mathcal{W}|$, and let $C$ denote a regular neighborhood of $\mathcal{A}_{\mathcal{W}}$ in $W$. We shall say that $\mathcal{W}$ is normal if (i) $W$ is a simple 3 -manifold, and (ii) $\overline{W-C}$ is ambiently isotopic in $W$ to the characteristic submanifold of the pair $(W, \partial W)$.

Proposition 4.7. Let $W$ be a simple 3-manifold. Let $\Sigma$ denote the characteristic submnanifold of the pair $(W, \partial W)$, and suppose that $\chi(\overline{W-\Sigma}) \geq 0$. Then $\overline{W-\Sigma}$ is a regular neighborhood of a properly embedded submanifold $\mathcal{A}$ of $W$, each component of which is an annulus. Furthermore, there is a normal book of I-bundles $\mathcal{W}$ such that $|\mathcal{W}|=W$ and $\mathcal{A}_{\mathcal{W}}=\mathcal{A}$.

Proof. Set $\mathcal{C}=\overline{W-\Sigma}$. As we observed in 4.5, the components of the frontier of $\Sigma$ are $\pi_{1}$-injective annuli. In particular, no component of $\partial \mathcal{C}$ is a 2 -sphere. Since $\chi(\mathcal{C}) \geq 0$, it follows that every component of $\partial \mathcal{C}$ is a torus. On the other hand, property (2) of $\Sigma$ stated in 4.5 implies that $\mathcal{C}$ is $\pi_{1}$-injective in $W$. Since $W$ is simple, it follows that the fundamental group of a component of $\mathcal{C}$ cannot have a rank-2 free abelian subgroup. Hence the components of $\mathcal{C}$ are boundary-reducible, and in view of the irreducibility of $W$ they must be solid tori.

Let $C$ be any component of $\mathcal{C}$. The frontier components of $C$ are among the frontier components of $\Sigma$, and we observed in 4.5 that these are essential annuli in $W$. In particular, it follows that the components of $C \cap \partial W$ are $\pi_{1^{-}}$ injective annuli on the solid torus $C$. Hence $C$ may be given the structure of a Seifert fibered space in such a way that $C \cap \partial W$ is saturated. It now follows from property (4) of $\Sigma$ that $C$ is ambiently isotopic in $W$ to a submanifold of $\Sigma$. Since $C$ is also ambiently isotopic to a submanifold of $W-\Sigma$, it must be ambiently isotopic to the regular neighborhood of a frontier component of $\Sigma$. This proves:

4.7.1. $\mathcal{C}$ is a regular neighborhood of a properly embedded 2-manifold $\mathcal{A} \subset$ $W$ whose components are annuli. In particular, each component of $\mathcal{C}$ has two frontier annuli. 
In particular, this gives the first assertion of the proposition.

Let $\Sigma_{0}$ denote the union of all components of $\Sigma$ that are solid tori, and set $\Sigma_{-}=\Sigma-\Sigma_{0}$. Since every component of $\Sigma$ is a solid torus or an $I$-bundle $P$ with $P \cap \partial W=\partial_{h} P$, each component of $\Sigma_{-}$is an $I$-bundle $P$ over a surface of negative Euler characteristic with $P \cap \partial W=\partial_{h} P$.

We now claim:

4.7.2. Each component of $\mathcal{C}$ has one frontier annulus contained in $\Sigma_{0}$ and one contained in $\Sigma_{-}$.

Let $C$ be any component of $\mathcal{C}$. According to 4.7.1, $C$ has two frontier annuli $A_{1}$ and $A_{2}$. Let $Q_{i}$ denote the component of $\Sigma$ containing $A_{i}$ (where a priori we might have $Q_{1}=Q_{2}$ ). To prove 4.7.2 we must show that $Q_{1}$ and $Q_{2}$ cannot both be contained in $\Sigma_{-}$or both be contained in $\Sigma_{0}$.

First suppose that the $Q_{i}$ are both contained in $\Sigma_{-}$. Then $Q \doteq Q_{1} \cup C \cup$ $Q_{2}$ may be given the structure of an $I$-bundle over a surface in such a way that $\partial_{h} Q=Q \cap \partial W$. It therefore follows from property (4) of $\Sigma$ stated in 4.5 that $Q$ is ambiently isotopic to a submanifold of $\Sigma$. But since $\chi\left(Q_{i}\right)<0$ for $i=1,2$ we have $\bar{\chi}(Q)>\bar{\chi}\left(Q_{i}\right)$ for $i=1,2$. Hence $Q$ cannot be ambiently isotopic to a submanifold of $Q_{i}$ for $i=1,2$. Furthermore, if $Q^{\prime}$ is a component of $\Sigma$ distinct from $Q_{1}$ and $Q_{2}$, then since $Q \cap Q^{\prime}=\emptyset$ and $\chi(Q)<0$, the $I$-bundle $Q$ cannot be ambiently isotopic to a submanifold of $Q^{\prime}$. This is a contradiction.

Now suppose that the $Q_{i}$ are both contained in $\Sigma_{0}$. Then the $Q_{i}$ are solid tori, and the image of the inclusion homomorphism $\pi_{1}\left(A_{i}\right) \rightarrow \pi_{1}\left(Q_{i}\right)$ has some finite index $m_{i}$ in $\pi_{1}\left(Q_{i}\right)$ for $i=1,2$. The fundamental group of $Q \doteq Q_{1} \cup C \cup Q_{2}$ has presentation $\left\langle x_{1}, x_{2}: x_{1}^{m_{1}}=x_{2}^{m_{2}}\right\rangle$. But since $W$ is simple and the frontier annuli of $Q$ are essential, $\pi_{1}(Q)$ has no rank-2 free abelian subgroup. Hence at least one of the $m_{i}$ must be equal to 1 , and we may assume that $m_{2}=1$. But this implies that $Q_{2}$ is ambiently isotopic to a submanifold of $Q_{1}$, which contradicts property (3) of $\Sigma$ stated in 4.5. This completes the proof of 4.7.2.

It follows from 4.7.1 and 4.7.2 that $\mathcal{B} \doteq \Sigma_{0} \cup \mathcal{C}$ is a regular neighborhood of $\Sigma_{0}$ in $W$, and that the frontier $\mathcal{A}^{\prime}$ of $\mathcal{B}$ is ambiently isotopic to $\mathcal{A}$. If we set $\mathcal{P}=\Sigma_{-}$, it follows from the definition given in $[6,5.1]$ that $\mathcal{W}^{\prime}=(W, \mathcal{B}, \mathcal{P})$ is a book of $I$-bundles. Normality is immediate from the construction. Since $\mathcal{A}_{\mathcal{W}^{\prime}}=\mathcal{A}^{\prime}$ is ambiently isotopic to $\mathcal{A}$, there is a normal book of $I$-bundles $\mathcal{W}$ with $|\mathcal{W}|=\left|\mathcal{W}^{\prime}\right|=W$ and $\mathcal{A}_{\mathcal{W}}=\mathcal{A}$.

Requiring that a book of $I$-bundles structure be normal rules out certain degeneracies. For example, if $W^{b}$ is an $I$-bundle over a closed surface of 
negative Euler characteristic, we may write $W^{b}=\left|\mathcal{W}^{b}\right|$ for some book of $I$ bundles $\mathcal{W}^{b}$ with $\mathcal{B}_{\mathcal{W}^{b}} \neq \emptyset$. Such a book of $I$-bundles $\mathcal{W}^{b}$ is not normal, and the following proposition would become false if the normal book of $I$-bundles $\mathcal{W}$ were replaced by $\mathcal{W}^{b}$.

Proposition 4.8. Let $\mathcal{W}$ be a normal book of I-bundles, and suppose that $A$ is an essential annulus in $|\mathcal{W}|$. Then $A$ is ambiently isotopic in $|\mathcal{W}|$ to either a vertical annulus in a page of $\mathcal{W}$, or an annulus contained in a binding.

Proof. Set $W=|\mathcal{W}|$. Let $\mathcal{C}$ be a regular neighborhood of $\mathcal{A}_{\mathcal{W}}$ in $W$. The definition of normality implies that (up to isotopy) $\Sigma \doteq \overline{W-\mathcal{C}}$ is the characteristic submanifold of $W$ relative to $\partial W$.

Let $V$ be a regular neighborhood of $A$ in $W$. Then $V$ may be given the structure of a Seifert fibered space in such a way that $V \cap \partial W$ is a saturated 2-manifold in $\partial V$. The components of the frontier of $V$ in $W$ are essential annuli in $W$. Hence property (4) of $\Sigma$ stated in 4.5 implies that $V$ is ambiently isotopic in $W$ to a submanifold of $\Sigma$. In particular, $A$ is ambiently isotopic in $W$ to an annulus $A^{\prime} \subset W-\mathcal{A}_{\mathcal{W}}$. Thus $A^{\prime}$ is contained in either a page or a binding of $W$.

If $A^{\prime}$ is contained in a page $P$, then $P$ is an $I$-bundle over a surface. Since $A$ is essential in $W$ (relative to $\partial W$ ), it is in particular essential in $P$ relative to $\partial_{h} P$. It therefore follows from Proposition 4.4 that $A^{\prime}$ is isotopic in $P$, by an ambient isotopy of $P$ which fixes $\partial_{v} P$, to a vertical annulus. The conclusion of the proposition follows.

In the lemma below, recall that if $K$ is a polyhedron then by definition $\bar{\chi}(K)=-\chi(K)$.

Lemma 4.9. Let $\mathcal{W}$ be a normal book of $I$-bundles. Let $Y$ be a compact 3dimensional submanifold of $|\mathcal{W}|$. Suppose that the following conditions hold:

(1) Each component of the frontier of $Y$ in $|\mathcal{W}|$ is an essential properly embedded annulus in $|\mathcal{W}|$.

(2) The 2-manifold $Y \cap \partial|\mathcal{W}|$ has two components $Z_{0}$ and $Z_{1}$, with $\bar{\chi}\left(Z_{0}\right)$ $=\bar{\chi}\left(Z_{1}\right)=1$.

(3) The inclusion homomorphism $H_{1}\left(Z_{0} ; \mathbb{Z}_{2}\right) \rightarrow H_{1}\left(Y ; \mathbb{Z}_{2}\right)$ is injective.

(4) For every solid torus $L \subset Y$, such that $L \cap Z_{0}$ is an annulus which is homotopically nontrivial in $|\mathcal{W}|$, the inclusion homomorphism $H_{1}\left(L \cap Z_{0} ; \mathbb{Z}\right) \rightarrow H_{1}(L, \mathbb{Z})$ is surjective. 
Then the inclusion homomorphism $\pi_{1}\left(Z_{0}\right) \rightarrow \pi_{1}(Y)$ is surjective.

Proof. We set $W=|\mathcal{W}|, \mathcal{P}=\mathcal{P}_{\mathcal{W}}$ and $\mathcal{B}=\mathcal{B}_{\mathcal{W}}$.

Since the frontier components of $Y$ relative to $W$ are annuli, we have

$$
\bar{\chi}(Y)=\frac{1}{2} \bar{\chi}(\partial Y)=\frac{1}{2}\left(\bar{\chi}\left(Z_{0}\right)+\bar{\chi}\left(Z_{1}\right)\right)=1 .
$$

By Proposition 4.8 we may assume that each frontier component of $Y$ is either a vertical annulus contained in $\mathcal{P}$ and disjoint from $\mathcal{A}$, or an annulus in $\mathcal{B}$. Then each component of $Y \cap \mathcal{P}$ is an $I$-sub-bundle of a page of $\mathcal{W}$, whose frontier is a disjoint union of vertical annuli in the page; each of these vertical annuli is either contained in, or disjoint from, the vertical boundary of the page. Furthermore, each component of $Y \cap \mathcal{B}$ is a solid torus in a binding, whose frontier is a disjoint union of $\pi_{1}$-injective annuli in the binding. In particular we have $\bar{\chi}(C) \geq 0$ for every component $C$ of $Y \cap \mathcal{P}$, and $\bar{\chi}(C)=0$ for every component $C$ of $Y \cap \mathcal{B}$.

Since the frontier components of $Y \cap \mathcal{B}$ and $Y \cap \mathcal{P}$ relative to $Y$ are annuli, we have

$$
1=\bar{\chi}(Y)=\bar{\chi}(Y \cap \mathcal{B})+\bar{\chi}(Y \cap \mathcal{P})=\bar{\chi}(Y \cap \mathcal{P})=\sum_{U} \bar{\chi}(U),
$$

where $U$ ranges over the components of $Y \cap \mathcal{P}$. Hence there is a component $U_{1}$ of $Y \cap \mathcal{P}$ with $\bar{\chi}\left(U_{1}\right)=1$, and $\bar{\chi}(U)=0$ for every component $U \neq U_{1}$ of $Y \cap \mathcal{P}$.

Since $U_{1}$ is a sub-bundle of a page of $\mathcal{W}$, it is an $I$-bundle over a surface $S$ with $\bar{\chi}(S)=1$, and the frontier of $U_{1}$ relative to $W$ is its vertical boundary.

We set $\mathcal{K}=\overline{Y-U_{1}}$.

Each component of the frontier of $U_{1}$ or $\mathcal{K}$ relative to $W$ is a component of either the frontier $\mathcal{A}_{\mathcal{W}}$ of $\mathcal{P}_{\mathcal{W}}$ relative to $W$, or the frontier of $Y$ relative to $W$. According to [6, Lemma 5.2], the components of $\mathcal{A}_{\mathcal{W}}$ are $\pi_{1}$-injective annuli in $W$. The components of the frontier of $Y$ are $\pi_{1}$-injective by hypothesis. Hence:

4.9.1. Each component of the frontier of $U_{1}$ or $\mathcal{K}$ relative to $W$ is a $\pi_{1}$ injective annulus in $W$.

The horizontal boundary of $U_{1}$ is a two-sheeted covering space of $S$, which is connected if and only if $U_{1}$ is a twisted $I$-bundle. In particular we have $\bar{\chi}\left(\partial_{h} U_{1}\right)=2 \bar{\chi}(S)=2$. On the other hand, we have $\partial_{h} U_{1}=$ $U_{1} \cap \partial W \subset Y \cap \partial W=Z_{0} \cup Z_{1}$. Hence $\partial_{h} U_{1} \subset Z_{j}$ for some $j \in\{0,1\}$. 
It follows from 4.9.1 that $\partial_{h} U_{1}$ is $\pi_{1}$-injective in $W$ and hence in $Z_{0} \cup Z_{1}$. Since $\bar{\chi}\left(Z_{0}\right)=\bar{\chi}\left(Z_{1}\right)=1$, the surface $\partial_{h} U_{1}$ cannot be contained in $Z_{0}$ or in $Z_{1}$. Hence:

4.9.2. $U_{1}$ is a trivial I-bundle over $S$, and its horizontal boundary has one component contained in $Z_{0}$ and one in $Z_{1}$.

For $i=1,2$, let us denote by $\Delta_{i}$ the component of $\partial_{h} U_{1}$ contained in $Z_{i}$.

Since $W$ is simple by the definition of a normal book of $I$-bundles, it follows from 4.9.1 that each component of $\mathcal{K}$ is simple. If $V$ is any component of $\mathcal{K}$, the components of $V \cap \mathcal{P}$ are components of $Y \cap \mathcal{P}$ distinct from $U_{1}$, and the components of $V \cap \mathcal{B}$ are components of $Y \cap \mathcal{B}$. Hence all components of $V \cap \mathcal{P}$ and $V \cap \mathcal{B}$ have Euler characteristic 0 . As the components of $(V \cap \mathcal{P}) \cap(V \cap \mathcal{B})$ are annuli it follows that $\chi(V)=0$. But the only simple 3 -manifold with nonempty boundary having Euler characteristic 0 is a solid torus. This shows:

4.9.3. Every component of $\mathcal{K}$ is a solid torus.

Let us consider any component $A$ of $U_{1} \cap \mathcal{K}$, and let $V$ denote the component of $\mathcal{K}$ containing $A$. According to 4.9.3, $V$ is a solid torus. Since $A$ is a component of the frontier of $U_{1}$ in $W$, it is a $\pi_{1}$-injective annulus in $W$ by 4.9.1, and hence in $V$. On the other hand, it follows from 4.9.2 that some component $c$ of $\partial A$ is contained in $Z_{0}$. A small nonambient isotopy of $V$ gives a solid torus $L$ such that

$L \cap Z_{0}$ is a regular neighborhood $R$ of $c$. Since $A$ is $\pi_{1}$-injective in $W$, the annulus $R$ is homotopically nontrivial in $W \mid$. Hypothesis (4.9) then implies that the inclusion homomorphism $H_{1}\left(L \cap Z_{0} ; \mathbb{Z}\right) \rightarrow H_{1}(L, \mathbb{Z})$ is an isomorphism, and hence that the inclusion homomorphism $\pi_{1}(A) \rightarrow \pi_{1}(V)$ is an isomorphism. This shows:

4.9.4. For every component $A$ of $U_{1} \cap \mathcal{K}$, if $V$ denotes the component of $\mathcal{K}$ containing $A$, the inclusion homomorphism $\pi_{1}(A) \rightarrow \pi_{1}(V)$ is an isomorphism.

Now suppose that $A_{1}$ and $A_{2}$ are two components of $U_{1} \cap \mathcal{K}$ contained in a single component $V$ of $\mathcal{K}$. For $i=1,2$ it follows from 4.9.2 that some component $c_{i}$ of $\partial A_{i}$ is contained in $Z_{0}$. Since $c_{1}$ and $c_{2}$ are disjoint, nontrivial simple closed curves on the torus $\partial V \subset W$, they represent the same element of $H_{1}\left(W ; \mathbb{Z}_{2}\right)$. Since by hypothesis $(4.9)$ the inclusion homomorphism $H_{1}\left(Z_{0} ; \mathbb{Z}_{2}\right) \rightarrow H_{1}\left(Y ; \mathbb{Z}_{2}\right)$ is injective, $c_{1}$ and $c_{2}$ cobound a subsurface 
$Q$ of $Z_{0}$. Since $c_{1}$ and $c_{2}$ are homotopically nontrivial, $Q$ is $\pi_{1}$-injective in $Z_{0}$, and hence $\bar{\chi}(Q) \leq \bar{\chi}\left(Z_{0}\right)=1$. As $Q$ is orientable and has exactly two boundary curves, it must be an annulus. On the other hand, $Q$ and $\Delta_{0}$ are subsurfaces of $Z_{0}$, and we have $\partial Q \subset \partial \Delta_{0}$. Hence either $\Delta_{0} \subset A$ or $A$ is a component of $\overline{Z_{0}-\Delta_{0}}$. But it follows from 4.9.1 that $\Delta_{0}$ is $\pi_{1}$-injective in $W$ and hence in $Z_{0}$; since $\bar{\chi}(\Delta)=1$, we cannot have $\Delta_{0} \subset A$. Thus we have proved:

4.9.5. If $A^{(0)}$ and $A^{(1)}$ are two components of $U_{1} \cap \mathcal{K}$ contained in the same component of $\mathcal{K}$, then some component of $\overline{Z_{0}-\Delta_{0}}$ is an annulus $Q$ having one boundary component contained in $\partial A^{(0)}$ and one contained in $\partial A^{(1)}$.

In particular, if $Q$ is an annulus having the properties stated in 4.9.5, then $\partial Q \subset \partial \Delta_{0}$. Since $\bar{\chi}\left(\Delta_{0}\right)=1$, the surface $\Delta_{0}$ has at most three boundary curves. Hence there is at most one unordered pair $\left\{A^{(0)}, A^{(1)}\right\}$ of components of $U_{1} \cap \mathcal{K}$ such that $A^{(0)}$ and $A^{(1)}$ are contained in the same component of $\mathcal{K}$. Equivalently:

4.9.6. There is no component of $\mathcal{K}$ whose boundary contains more than two components of $U_{1} \cap \mathcal{K}$, and there is at most one component of $\mathcal{K}$ whose boundary contains two components of $U_{1} \cap \mathcal{K}$.

In view of 4.9.6, the argument now divides into two cases.

Case I: The boundary of each component of $\mathcal{K}$ contains only one component of $U_{1} \cap \mathcal{K}$. In this case, let $A_{1}, \ldots, A_{m}$ denote the components of $U_{1} \cap \mathcal{K}$. (Since $\bar{\chi}(S)=1$ we have $m \leq 3$.) Let $V_{i}$ denote the component of $\mathcal{K}$ containing $A_{i}$; thus the solid tori $V_{1}, \ldots, V_{m}$ are all distinct. We have $Y=$ $U_{1} \cup V_{1} \cup \cdots \cup V_{m}$. By 4.9.4 the inclusion homomorphism $\pi_{1}\left(A_{i}\right) \rightarrow \pi_{1}\left(V_{i}\right)$ is an isomorphism for $i=1, \ldots, m$. Hence the inclusion homomorphism $\pi_{1}\left(U_{1}\right) \rightarrow \pi_{1}(Y)$ is an isomorphism. But by 4.9 .2 , the inclusion homomorphism $\pi_{1}\left(\Delta_{0}\right) \rightarrow \pi_{1}\left(U_{1}\right)$ is an isomorphism. Hence the inclusion homomorphism $\pi_{1}\left(\Delta_{0}\right) \rightarrow \pi_{1}(Y)$ is an isomorphism, and in particular the inclusion homomorphism $\pi_{1}\left(Z_{0}\right) \rightarrow \pi_{1}(Y)$ is surjective. This establishes the conclusion of the lemma in this case.

Case II: There is a component $V_{0}$ of $\mathcal{K}$ whose boundary contains more than one component of $U_{1} \cap \mathcal{K}$. By 4.9.6, this component $V_{0}$ is unique and contains exactly two components of $U_{1} \cap \mathcal{K}$, which we denote $A_{0}^{(0)}$ and $A_{0}^{(1)}$.

According to $4.9 .3, V_{0}$ is a solid torus, and according to 4.9 .4 the inclusion homomorphism $\pi_{1}\left(A_{0}^{(i)}\right) \rightarrow \pi_{1}\left(V_{0}\right)$ is an isomorphism for $i=0,1$. Hence 
there is a homeomorphism $h: V_{0} \rightarrow S^{1} \times[0,1] \times[0,1]$ such that $h\left(A_{0}^{(i)}\right)=$ $S^{1} \times\{i\} \times[0,1]$ for $i=0,1$. This allows us to extend the $I$-bundle structure of $U_{1}$ to an $I$-bundle structure for $L \doteq U_{1} \cup V_{0}$. (Note, however, that the horizontal boundary of $L$ need not be contained in $\partial W$.)

According to 4.9.5, some component of $\overline{Z_{0}-\Delta_{0}}$ is an annulus $Q$ having one boundary component contained in $\partial A^{(0)}$ and one contained in $\partial A^{(1)}$. This implies that the $I$-bundle $L$ is trivial, and that one of its horizontal boundary components, which we shall denote by $\Theta$, is contained in $Z_{0}$.

Now let $A_{1}, \ldots, A_{m}$ denote the components of $U_{1} \cap \mathcal{K}$ distinct from $A_{0}^{(0)}$ and $A_{0}^{(1)}$. (Since $\bar{\chi}(S)=1$ one may show that $m \leq 1$, but this will not be used.) Let $V_{i}$ denote the component of $\mathcal{K}$ containing $A_{i}$; thus the solid tori $V_{1}, \ldots, V_{m}$ are all distinct. We have $Y=L \cup V_{1} \cup \cdots \cup V_{m}$. By 4.9.4 the inclusion homomorphism $\pi_{1}\left(A_{i}\right) \rightarrow \pi_{1}\left(V_{i}\right)$ is an isomorphism for $i=1, \ldots, m$. Hence the inclusion homomorphism $\pi_{1}(L) \rightarrow \pi_{1}(Y)$ is an isomorphism. But since $L$ is a trivial $I$-bundle and $\Theta$ is one component of its horizontal boundary, the inclusion homomorphism $\pi_{1}(\Theta) \rightarrow \pi_{1}(L)$ is an isomorphism. Hence the inclusion homomorphism $\pi_{1}(\Theta) \rightarrow \pi_{1}(Y)$ is an isomorphism, and in particular the inclusion homomorphism $\pi_{1}\left(Z_{0}\right) \rightarrow \pi_{1}(Y)$ is surjective. Thus the conclusion of the lemma is established in this case as well.

\section{Trimonic manifolds}

The kind of object discussed in this section, defined in 5.7 below, is obtained from a trivial $I$-bundle over a surface $S$ by first attaching a single 1-handle $\alpha$ to one boundary component, then attaching a single 2 -handle whose boundary runs thrice over $\alpha$. Here we prove that such a trimonic manifold has incompressible boundary (in Lemma 5.13) and is not the underlying space of a normal book of $I$-bundles (Lemma 5.14), if it is nondegenerate (cf. Definition 5.10).

5.1. Let $V$ be a point of an oriented surface $S$. By an ordered triod based at $V$ we shall mean an ordered triple $\left(A_{0}, A_{1}, A_{2}\right)$ of closed topological arcs in $S$, each having $V$ as an endpoint, such that $A_{i} \cap A_{j}=\{V\}$ whenever $i \neq j$.

Suppose that $\left(A_{0}, A_{1}, A_{2}\right)$ is an ordered triod based at $V$. For $i=0,1,2$ let $x_{i}$ denote the endpoint of $A_{i}$ that is distinct from $V$. Then there is a disk $\delta \subset S$ such that $A_{0} \cup A_{1} \cup A_{2} \subset \delta$ and $\left(A_{0} \cup A_{1} \cup A_{2}\right) \cap \partial \delta=\left\{x_{0}, x_{1}, x_{2}\right\}$. We shall express this by saying that the triod $\left(A_{0}, A_{1}, A_{2}\right)$ is properly embedded in $\delta$. The orientation of $S$ restricts to an orientation of $\delta$, which in turn induces an orientation of $\partial \delta$. We shall say that the ordered triod $\left(A_{0}, A_{1}, A_{2}\right)$ 
is positive if the ordered triple $\left(x_{0}, x_{1}, x_{2}\right)$ is in counterclockwise order on $\partial \delta$, and negative otherwise.

5.2. Suppose that $\theta$ is an oriented open arc. We denote by $\theta^{\prime}$ the same arc with the opposite orientation. By a terminal segment of $\theta$ we mean a subset $A$ of $\theta$ which has the form $h((t, 1))$ for some orientation-preserving homeomorphism $h:(0,1) \rightarrow \theta$ and some point $t \in(0,1)$. By an initial segment of $\theta$ we mean a terminal segment of $\theta^{\prime}$.

5.3. Now suppose that $\Gamma$ is a graph (i.e. a 1-dimensional $\mathrm{CW}$ complex) contained in an oriented surface $S$. By an oriented edge of $\Gamma$ we mean simply an (open) edge which is equipped with an orientation. Let $V$ be a vertex of $\Gamma$, and let $\left(e_{0}, e_{1}, e_{2}\right)$ be an ordered triple of oriented edges of $\Gamma$ with terminal vertex $V$. Assume that the $e_{i}$ are distinct as oriented edges (although two of them may be opposite orientations of the same underlying edge). We may choose terminal segments $A_{i}$ of the $e_{i}$ in such a way that $\left(\bar{A}_{0}, \bar{A}_{1}, \bar{A}_{2}\right)$ is an ordered triod in $S$. We shall say that the ordered triple $\left(e_{0}, e_{1}, e_{2}\right)$ is positive if the ordered triod $\left(\bar{A}_{0}, \bar{A}_{1}, \bar{A}_{2}\right)$ is positive, and negative otherwise.

5.4. Let $Z$ be a planar surface with three boundary curves, and let $\star \in$ int $Z$ be a base point. An ordered pair $\left(z_{1}, z_{2}\right)$ of elements of $\pi_{1}(Z, \star)$ will be called a geometric basis for $\pi_{1}(Z, \star)$ if the boundary curves of $Z$ may be indexed as $\left(C_{i}\right)_{1 \leq i \leq 3}$ in such a way that for $i=1,2,3$ there exist a point $p_{i} \in C_{i}$, a closed path $\gamma_{i}$ in $C_{i}$ based at $p_{i}$ and an oriented (embedded) arc $\tau_{i}$ from $\star$ to $p_{i}$, such that

- the $\tau_{i}$ have pairwise disjoint interiors;

- $\tau_{i} \cap C_{i}=\left\{p_{i}\right\}$ for each $i$;

- $\left[\gamma_{i}\right]$ generates $\pi_{1}\left(C_{i}, p_{i}\right)$ for each $i$;

- $z_{i}=\left[\tau_{i} * \gamma_{i} * \overline{\tau_{i}}\right]$ for $i=1,2$; and

- $z_{1}^{-1} z_{2}=\left[\tau_{3} * \gamma_{3} * \overline{\tau_{3}}\right]$.

The constituent parts of a geometric basis are pictured in figure 5.1. Note that if $\left(z_{1}, z_{2}\right)$ is a geometric basis for $\pi_{1}(Z, \star)$ then $z_{1}$ and $z_{2}$ freely generate $\pi_{1}(Z, \star)$. 


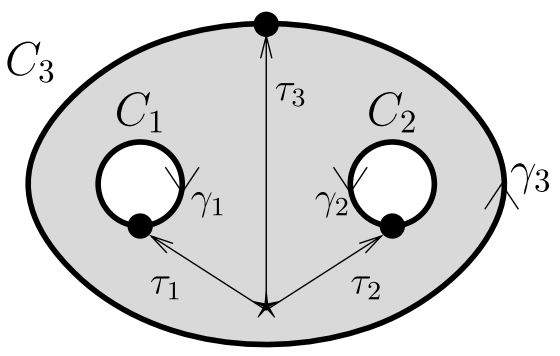

Figure 5.1: A geometric basis.

5.5. Let $\Gamma$ be a theta graph contained in an oriented surface $S$. Let $W$ and $V$ denote the vertices of $\Gamma$, and let $\beta_{0}, \beta_{1}$ and $\beta_{2}$ denote the oriented edges having initial vertex $W$ and terminal vertex $V$. Suppose that the ordered triple $\left(\beta_{0}, \beta_{1}, \beta_{2}\right)$ of edges terminating at $V$ is positive, and that the ordered triple $\left(\beta_{0}^{\prime}, \beta_{1}^{\prime}, \beta_{2}^{\prime}\right)$ of oriented edges terminating at $W$ is negative. Then a regular neighborhood $Z$ of $\Gamma$ in $S$ is a planar surface with three boundary curves, and $\left(\left[\beta_{0}^{\prime} * \beta_{1}\right],\left[\beta_{0}^{\prime} * \beta_{2}\right]\right)$ is a geometric basis of $\pi_{1}(Z, V)$.

5.6. Let $\Gamma$ be an eyeglass graph contained in an oriented surface $S$. Let $W$ and $V$ denote the vertices of $\Gamma$, let $\beta_{0}$ denote the oriented edge having initial vertex $W$ and terminal vertex $V$, and let $\beta_{1}$ and $\beta_{2}$ be oriented loops based at $W$ and $V$ respectively. Suppose that the ordered triple $\left(\beta_{0}, \beta_{2}^{\prime}, \beta_{2}\right)$ of edges terminating at $V$ is positive, and that the ordered triple $\left(\beta_{0}^{\prime}, \beta_{1}^{\prime}, \beta_{1}\right)$ of edges terminating at $W$ is negative. Then a regular neighborhood $Z$ of $\Gamma$ in $S$ is a planar surface with three boundary curves, and $\left(\left[\beta_{2}\right],\left[\beta_{0}^{\prime} * \beta_{1} * \beta_{0}\right]\right)$ is a geometric basis of $\pi_{1}(Z, V)$.

Definition 5.7. Let $X$ be a compact orientable 3-manifold, and let $S$ be a component of $\partial X$. We shall say that $X$ is a trimonic manifold relative to $S$ if there exists a properly embedded arc $\alpha \subset X$ and a PL map $f$ of a PL 2-disk $D$ into $X$, such that the following conditions hold:

(1) $f^{-1}(\alpha)$ is a union of three disjoint arcs in $\partial D$;

(2) $f$ maps each component of $f^{-1}(\alpha)$ homeomorphically onto $\alpha$;

(3) $f \mid\left(\right.$ int $\left.D \cup\left((\partial D)-f^{-1}(\alpha)\right)\right)$ is one-to-one;

(4) $f(\operatorname{int} D) \subset \operatorname{int} X$;

(5) $f\left((\partial D)-f^{-1}(\alpha)\right) \subset S$;

(6) $X$ is a semi-regular neighborhood of $S \cup f(D)$. 
By a semi-regular neighborhood of $K$ in $M$ we mean a neighborhood of $K$ which is a compact PL submanifold of $M$ and admits a polyhedral collapse to $K$, but does not necessarily meet the boundary of $M$ regularly.

Note that condition (5.7) implies that the endpoints of $\alpha$ lie in $S$.

A PL map $f$ of a 2-disk $D$ into $X$ such that (5.7)-(5.7) hold for some properly embedded arc $\alpha$ in $X$ will be called a defining hexagon for the trimonic manifold $X$ relative to $S$.

Notation 5.8. Suppose that $f: D \rightarrow X$ is a defining hexagon for a trimonic manifold $X$ relative to $S$. Note that the arc $\alpha$ appearing in Definition 5.7 is uniquely determined by $D$. We shall denote this arc by $\alpha_{f}$. Furthermore, we shall denote by $\Gamma_{f}$ the PL set $f\left((\partial D)-f^{-1}(\alpha)\right)=f(D) \cap \partial X \subset S$.

Lemma 5.9. Suppose that $X$ is a trimonic manifold relative to $S$. Let $f: D \rightarrow X$ be a defining hexagon for $X$, and set $\alpha=\alpha_{f}$ and $\Gamma=\Gamma_{f}$. Then $\Gamma$ is homeomorphic to either a theta graph (the "theta case") or an eyeglass graph (the "eyeglass case"), and a regular neighborhood $Z$ of $\Gamma$ in $S$ is a planar surface with three boundary curves. Furthermore, for some (and hence for any) base point $\star \in \operatorname{int} Z$, there is an ordered basis $\left(t, u_{1}, u_{2}\right)$ of the rank-3 free group $\pi_{1}(Z \cup \alpha, \star)$ with the following properties (see figure 5.2 ):

- the inclusion homomorphism $\pi_{1}(Z, \star) \rightarrow \pi_{1}(Z \cup \alpha, \star)$ maps some geometric basis of $\pi_{1}(Z, \star)$ to the pair $\left(u_{1}, u_{2}\right)$; and

- $\partial D$ may be oriented so that the conjugacy class in $\pi_{1}(Z \cup \alpha, \star)$ represented by $f \mid \partial D: \partial D \rightarrow Z \cup \alpha$ is $t^{2} u_{1} t u_{2}$ in the theta case, or $t^{2} u_{1} t^{-1} u_{2}$ in the eyeglass case.

Proof. We denote the components of $f^{-1}(\alpha)$ by $a_{0}, a_{1}$ and $a_{2}$, and we denote the components of $\overline{(\partial D)-f^{-1}(\alpha)}$ by $b_{0}, b_{1}$ and $b_{2}$. We take the $a_{i}$ and $b_{i}$

Case (I)

$\phi_{2}$ preserves orientation

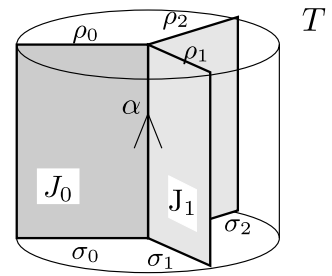

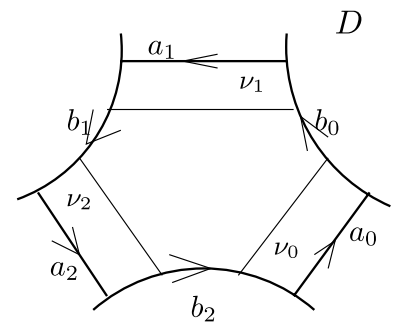

Case (II) $\phi_{2}$ reverses orientation

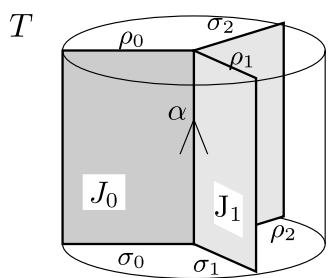

Figure 5.2: Some objects defined in the proof of Lemma 5.9. 
to be labeled in such a way that $a_{i}$ and $b_{j}$ share an endpoint if and only if $i$ is congruent to either $j$ or $j+1$ modulo 3 (see Figure 5.2). For $i=0,1,2$ we set $\beta_{i}=f\left(\operatorname{int} b_{i}\right)$. Then $\beta_{i}$ is an open arc in $S-\partial \alpha$, and $\overline{\beta_{i}}-\beta_{i} \subset \partial \alpha$. Hence $\Gamma=\overline{\beta_{0} \cup \beta_{1} \cup \beta_{2}}$ may be given the structure of a graph whose vertices are the endpoints of $\alpha$, and whose edges are $\beta_{0}, \beta_{1}$ and $\beta_{2}$.

Since $\alpha$ is an embedded arc, $f$ maps the terminal point of $\beta_{i}$ and the initial point of $\beta_{i+1}$ to distinct vertices of $\Gamma$. In particular, each vertex of $\Gamma$ has valence 3 . Since $\Gamma$ has three edges and two vertices, it is either a theta graph or an eyeglass graph.

For $i=0,1,2$ we denote by $P_{i}$ the common endpoint of the $\operatorname{arcs} a_{i}$ and $b_{i-1}$ in $\partial D$ (where subtraction is interpreted modulo 3 ), and by $Q_{i}$ the common endpoint of $a_{i}$ and $b_{i}$.

If we fix an orientation of $\partial D$ then each of the arcs $a_{i}$ and $b_{i}$ inherits an orientation. We choose the orientation of $\partial D$ in such a way that $P_{i}$ and $Q_{i}$ are, respectively, the initial and terminal points of $a_{i}$, while $Q_{i}$ and $P_{i+1}$ are, respectively, the initial and terminal points of $b_{i}$. According to Definition 5.7, $f$ restricts to a homeomorphism $\phi_{i}: a_{i} \rightarrow \alpha$ for $i=0,1,2$.

Let $T$ be a PL tubular neighborhood of $\alpha$ in $X$. We may choose $T$ in such a way that $f$ is transverse to the frontier of $T$ and $f^{-1}(T)$ is a regular neighborhood of $f^{-1}(\alpha)=a_{0} \cup a_{1} \cup a_{2}$ in $D$. For each $i \in\{0,1,2\}$, let $\nu_{i}$ denote the component of $f^{-1}(T)$ containing $a_{i}$. Then $\nu_{i} \cap \partial D$ has the form $a_{i} \cup s_{i} \cup r_{i}$, where $s_{i}$ is the closure of a terminal segment of $b_{i-1}$ and $r_{i}$ is the closure of an initial segment of $b_{i}$.

It follows from Definition 5.7 that $f$ maps each $\nu_{i}$ homeomorphically on to a PL disk $J_{i} \subset T$, and that $J_{i} \cap J_{j}=\alpha$ when $i \neq j$. The intersection of $T$ with $\partial X$ consists of two PL disks; for each $i \in\{0,1,2\}$, one of these disks meets $J_{i}$ in the $\operatorname{arc} \sigma_{i} \doteq f\left(s_{i}\right)$, and the other meets $J_{i}$ in the $\operatorname{arc} \rho_{i} \doteq f\left(r_{i}\right)$. Hence we may identify $T$ by a PL homemorphism with $\delta \times \alpha$, where $\delta$ is a PL disk, in such a way that $\alpha=\{o\} \times \alpha$ for some interior point $o$ of $\delta$; and so that for $i=0,1,2$ we have $J_{i}=t_{i} \times \alpha$ for some arc $t_{i} \subset \delta$. Each $t_{i}$ has one endpoint in $\partial \delta$ and one at $o$, and we have $t_{i} \cap t_{j}=o$ for $i \neq j$. The components of $t_{i} \times \partial \alpha$ are $\sigma_{i}$ and $\rho_{i}$.

We may orient $\alpha$ in such a way that at least two of the homeomorphisms $\phi_{i}: a_{i} \rightarrow \alpha$ are orientation-preserving. Hence, after a possible cyclic relabeling of the $a_{i}$ (and the $b_{i}$ ), we may assume that $\phi_{0}$ and $\phi_{1}$ are orientationpreserving.

We denote by $V$ and $W$, respectively, the initial and terminal endpoints of $\alpha$ with respect to the orientation that we have chosen. Thus for $i=0,1$ we have $f\left(P_{i}\right)=V$ and $f\left(Q_{i}\right)=W$. 
We shall distinguish two cases according to whether the homeomorphism $\phi_{2}: a_{2} \rightarrow \alpha$ (I) preserves or (II) reverses orientation. In case I we have $f\left(P_{2}\right)=V$ and $f\left(Q_{2}\right)=W$, while in case II we have $f\left(P_{2}\right)=W$ and $f\left(Q_{2}\right)=V$. Hence we may define ordered triods (see 5.1) $\mathcal{T}_{V}$ and $\mathcal{T}_{W}$ based at $V$ and $W$, respectively, by setting $\mathcal{T}_{V}=\left(\sigma_{0}, \sigma_{1}, \sigma_{2}\right)$ and $\mathcal{T}_{W}=\left(\rho_{0}, \rho_{1}, \rho_{2}\right)$ in case I, and $\mathcal{T}_{V}=\left(\sigma_{0}, \sigma_{1}, \rho_{2}\right)$ and $\mathcal{T}_{W}=\left(\rho_{0}, \rho_{1}, \sigma_{2}\right)$ in case II. In each case, if we denote by $\delta_{V}$ and $\delta_{W}$ the components of $T \cap \partial X$ containing $V$ and $W$, respectively, the triods $\mathcal{T}_{V}$ and $\mathcal{T}_{W}$ are properly embedded (see 5.1) in $\delta_{V}$ and $\delta_{W}$.

If we identify $T$ as above with $\delta \times \alpha$, we have $\delta_{V}=\delta \times\{V\}$ and $\delta_{W}=$ $\delta \times\{W\}$. We may then define a homeomorphism $\psi: \delta_{V} \rightarrow \delta_{W}$ by $\psi(x, V)=$ $(x, W)$.

We orient $S$ in such a way that $\mathcal{T}_{V}$ is a positive ordered triod (see 5.1). Each of the disks $\delta_{V}$ and $\delta_{W}$ inherits an orientation from $S$. The orientability of the 3-manifold $X$ implies that $\psi: \delta_{V} \rightarrow \delta_{W}$ is an orientation-reversing homeomorphism.

By the properties of the identification stated above, we have $\psi\left(\sigma_{i}\right)=\rho_{i}$ for $i=0,1$; and in Case I we have $\psi\left(\sigma_{2}\right)=\rho_{2}$, while in Case II we have $\psi\left(\rho_{2}\right)=\sigma_{2}$. Since $\mathcal{T}_{V}$ is a positive ordered triod, and since $\psi$ reverses orientation, the ordered triod $\mathcal{T}_{W}$ is negative.

We orient each open arc $\beta_{i}$ in such a way that $f \mid \operatorname{int} b_{i}: \operatorname{int} b_{i} \rightarrow \beta_{i}$ is orientation-preserving. Since $\alpha, \beta_{0}, \beta_{1}$ and $\beta_{2}$ are now equipped with orientations, their closures define elements of the fundamental groupoid $\Pi(f(D))$ which we denote by $[\alpha],\left[\beta_{0}\right],\left[\beta_{1}\right]$ and $\left[\beta_{2}\right]$. We let $c$ denote the closed path $a_{0} * b_{0} * a_{1} * b_{1} * a_{2} * b_{2}$ in $\partial D$, based at $P_{0}$. Then $[c]$ generates $\pi_{1}\left(\partial D, P_{0}\right)$. We set $\gamma=f \circ c$.

We have

$$
[\gamma]=[\alpha]\left[\beta_{0}\right][\alpha]\left[\beta_{1}\right][\alpha]^{\epsilon}\left[\beta_{2}\right] \in \pi_{1}(f(D), V) \subset \Pi(f(D)),
$$

where $\epsilon=1$ in Case I, and $\epsilon=-1$ in Case II.

To prove the conclusions of the lemma in Case I, we note that since $f\left(P_{i}\right)=V$ and $f\left(Q_{i}\right)=W$ for each $i$, each $\beta_{i}$ has $W$ as initial vertex and $V$ as terminal vertex. Thus $\Gamma$ is a theta graph, and Case I is the "theta case" referred to in the statement of the lemma.

Since $\mathcal{T}_{V}=\left(\sigma_{0}, \sigma_{1}, \sigma_{2}\right)$ is a positive ordered triod based at $V$, and the interior of $\sigma_{i}$ is a terminal segment of $\beta_{i-1}$, the triple $\left(\beta_{2}, \beta_{0}, \beta_{1}\right)$ of edges terminating at $V$ is positive in the sense of 5.3. Hence the triple $\left(\beta_{0}, \beta_{1}, \beta_{2}\right)$ is positive. Likewise, since $\mathcal{T}_{W}=\left(\rho_{0}, \rho_{1}, \rho_{2}\right)$ is a negative ordered triod based 
at $W$, and since the interior of $\rho_{i}$ is an initial segment of $\beta_{i}$ - and therefore a terminal segment of $\beta_{i}^{\prime}$ - the triple $\left(\beta_{0}^{\prime}, \beta_{1}^{\prime}, \beta_{2}^{\prime}\right)$ of edges terminating at $W$ is negative. It now follows from 5.5 that a regular neighborhood $Z$ of $\Gamma$ in $S$ is a planar surface with three boundary curves, and that if we set $z_{1}=\left[\beta_{0}^{\prime} * \beta_{1}\right]$ and $\left.z_{2}=\left[\beta_{0}^{\prime} * \beta_{2}\right]\right)$, then $\left(z_{1}, z_{2}\right)$ is a geometric basis of $\pi_{1}(Z, V)$.

In particular $\pi_{1}(Z, V)$ is freely generated by $z_{1}$ and $z_{2}$, and hence $\pi_{1}(Z \cup$ $\alpha, V)$ is generated by $t, u_{1}$ and $u_{2}$, where $u_{i}$ denotes the image of $z_{i}$ under the inclusion homomorphism $\pi_{1}(Z, V) \rightarrow \pi_{1}(Z \cup \alpha, V)$, and $t=\left[\alpha * \beta_{0}\right]$.

We have

$$
\begin{aligned}
{[\gamma] } & =\left[\alpha * \beta_{0} * \alpha * \beta_{1} * \alpha * \beta_{2}\right] \\
& =\left[\alpha * \beta_{0} * \alpha * \beta_{0} * \beta_{0}^{\prime} * \beta_{1} * \alpha * \beta_{0} * \beta_{0}^{\prime} * \beta_{2}\right] \\
& =t^{2} u_{1} t u_{2} .
\end{aligned}
$$

This establishes the conclusions of the lemma in the theta case.

In Case II we have $f\left(P_{i}\right)=V$ and $f\left(Q_{i}\right)=W$ for $i=0,1$, while $f\left(P_{2}\right)=$ $W$ and $f\left(Q_{2}\right)=V$. It follows that the closures of $\beta_{1}$ and $\beta_{2}$ are loops based at $W$ and $V$, respectively, whereas $\beta_{0}$ has initial point $W$ and terminal point $V$. Thus $\Gamma$ is an eyeglass graph, and Case II is the "eyeglass case" referred to above.

Since $\mathcal{T}_{V}=\left(\sigma_{0}, \sigma_{1}, \rho_{2}\right)$ is a positive ordered triod based at $V$, and since the interiors of $\sigma_{0}, \sigma_{1}$ and $\rho_{2}$ are, respectively, terminal segments of $\beta_{2}, \beta_{0}$ and $\beta_{2}^{\prime}$, the triple $\left(\beta_{2}, \beta_{0}, \beta_{2}^{\prime}\right)$ of edges terminating at $V$ is positive. Hence the triple $\left(\beta_{0}, \beta_{2}^{\prime}, \beta_{2}\right)$ is positive. Likewise, since $\mathcal{T}_{W}=\left(\rho_{0}, \rho_{1}, \sigma_{2}\right)$ is a negative ordered triod based at $W$, and since the interiors of $\rho_{0}, \rho_{1}$ and $\sigma_{2}$ are, respectively, terminal segments of $\beta_{0}^{\prime}, \beta_{1}^{\prime}$ and $\beta_{1}$, the triple $\left(\beta_{0}^{\prime}, \beta_{1}^{\prime}, \beta_{1}\right)$ of edges terminating at $W$ is negative. It now follows from 5.6 that a regular neighborhood $Z$ of $\Gamma$ in $S$ is a planar surface with three boundary curves, and that if we set $\left.z_{1}=\left[\beta_{0}^{\prime} * \beta_{1} * \beta_{0}\right]\right)$ and $z_{2}=\left[\beta_{2}\right]$, then $\left(z_{1}, z_{2}\right)$ is a geometric basis of $\pi_{1}(Z, V)$.

In particular $\pi_{1}(Z, V)$ is freely generated by $z_{1}$ and $z_{2}$, and hence $\pi_{1}(Z \cup$ $\alpha, V)$ is generated by $t, u_{1}$ and $u_{2}$, where $u_{i}$ denotes the image of $z_{i}$ under the inclusion homomorphism $\pi_{1}(Z, V) \rightarrow \pi_{1}(Z \cup \alpha, V)$, and $t=\left[\alpha * \beta_{0}\right]$.

We have

$$
\begin{aligned}
{[\gamma] } & =\left[\alpha * \beta_{0} * \alpha * \beta_{1} * \alpha^{\prime} * \beta_{2}\right] \\
& =\left[\alpha * \beta_{0} * \alpha * \beta_{0} * \beta_{0}^{\prime} * \beta_{1} * \beta_{0} * \beta_{0}^{\prime} * \alpha^{\prime} * \beta_{2}\right] \\
& =(t)^{2} u_{1}(t)^{-1} u_{2} .
\end{aligned}
$$

This establishes the conclusions of the lemma in the eyeglass case. 
Definition 5.10. Let $X$ be a trimonic manifold relative to $S$. We shall say that $X$ is nondegenerate if there is a defining hexagon $f: D \rightarrow X$ such that no component of $S-\Gamma_{f}$ is an open disk.

Lemma 5.11. Suppose that $X$ is a nondegenerate trimonic manifold relative to a component $S$ of $\partial X$. Then $H g(X) \leq 1+\operatorname{genus}(S)$. Furthermore, $X$ contains a compact connected 3-dimensional submanifold $Y$ with the following properties:

(1) Each component of the frontier of $Y$ in $X$ is an essential annulus in $X$, joining distinct components of $\partial X$.

(2) The 2-manifold $Y \cap \partial X$ has two components $Z_{0}$ and $Z_{1}$, where $Z_{0} \subset S$ and $Z_{1} \not \subset S$, and each $Z_{i}$ is a planar surface with three boundary curves.

(3) The group $\pi_{1}(Y)$ is free of rank 2.

(4) For any base point $\star \in \operatorname{int} Z_{0}$, there exists an ordered pair of generators $(x, y)$ of $\pi_{1}(Y, \star)$, such that either the pair $\left(x, y x^{-1} y^{2}\right)$ or the pair $\left(x, y^{-1} x^{-1} y^{2}\right)$ is the image of some geometric basis of $\pi_{1}\left(Z_{0}, \star\right)$ under the inclusion homomorphism $\pi_{1}\left(Z_{0}, \star\right) \rightarrow \pi_{1}(Y, \star)$.

(5) Each component $Q$ of $\overline{X-Y}$ may be given the structure of a trivial I-bundle over a 2-manifold, in such a way that $Y \cap Q$ is the vertical boundary of $Q$.

Proof. We fix a defining hexagon $f: D \rightarrow X$, and set $\alpha=\alpha_{f}$ and $\Gamma=\Gamma_{f}$. Since $X$ is nondegenerate, we may choose $f$ in such a way that:

5.11.1. No component of $S-\Gamma$ is an open disk.

Let $T$ be a PL tubular neighborhood of $\alpha$ in $X$, and let $\delta$ be a properly embedded disk in $T \cap \operatorname{int} X$ which crosses $\alpha$ transversally in one point. We may choose $T$ in such a way that $f^{-1}(T)$ is a regular neighborhood of $f^{-1}(\alpha)=a_{0} \cup a_{1} \cup a_{2}$ in $D$. Let $D^{\prime}$ denote the disk $\overline{D-f^{-1}(T)}$. Then the frontier of $D^{\prime}$ is the union of three arcs $a_{0}^{\prime}, a_{1}^{\prime}$ and $a_{2}^{\prime}$, where $a_{i}^{\prime}$ is the frontier in $D$ of the component of $f^{-1}(T)$ containing $a_{i}$.

According to Definition 5.7, $f$ maps each $a_{i}$ homeomorphically onto $\alpha$. Hence we may choose $\delta$ so that for each $i$, the arc $f\left(a_{i}^{\prime}\right)$ meets $\partial \delta$ transversally in exactly one point. In particular: 


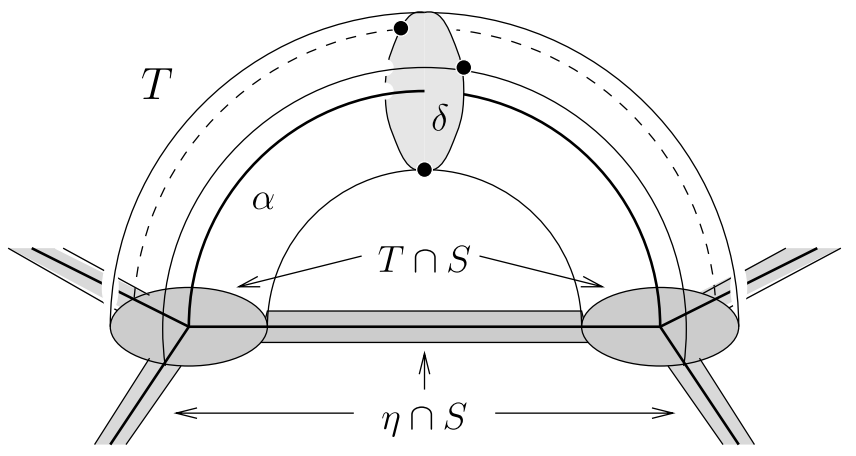

Figure 5.3: The shaded neighborhood is $K \cap S=(T \cap S) \cup(\eta \cap S)$. The graph $\Gamma \subset K \cap S$ is indicated in bold, as are the three points of $f\left(\partial D^{\prime}\right) \cap \partial \delta$.

5.11.2. The simple closed curve $f\left(\partial D^{\prime}\right)$ meets $\partial \delta$ in exactly three points, and these are transversal points of intersection within the frontier annulus of $T$.

Let $\eta$ denote a regular neighborhood in $\overline{X-T}$ of the properly embedded disk $f\left(D^{\prime}\right) \subset \overline{X-T}$. Then $K \doteq T \cup \eta$ is a regular neighborhood of $f(D)$ in $X$.

Let $\mathcal{C}=\Sigma \times I$, where $\Sigma$ is a surface homeomorphic to $S$, and attach $\mathcal{C}$ to $X$ by using such a homeomorphism to identify $\Sigma \times\{1\}$ with $S$. We note that the pair $(X, S)$ is homeomorphic to $\left(X \cup \mathcal{C}, S_{0}\right)$, where $S_{0} \doteq \Sigma \times\{0\} \subset$ $\partial(X \cup \mathcal{C})$. We set $X_{0}=\mathcal{C} \cup K \subset X \cup \mathcal{C}$. Since $K$ is a regular neighborhood of $f(D)$ in $X$, the manifold $X \cup \mathcal{C}$ is a semi-regular neighborhood of its 3 -dimensional submanifold $X_{0}$. It follows that the pair $\left(X_{0}, S_{0}\right)$ is homeomorphic to $\left(X \cup \mathcal{C}, S_{0}\right)$, and hence to $(X, S)$. It therefore suffices to show that the conclusions of the lemma are true when $X$ and $S$ are replaced by $X_{0}$ and $S_{0}$.

We first note that the manifold $V \doteq \mathcal{C} \cup T$ is a compression body with $\partial_{-} V=S_{0}$. If we set $g=\operatorname{genus}(S)$, the genus of $\partial_{+} V$ is $g+1$. In particular $V$ admits a Heegaard splitting of genus $g+1$. We have $X_{0}=V \cup \eta$, so that $X_{0}$ is obtained from $V$ by adding a 2-handle. It therefore follows from [6, Lemma 2.1] that $\operatorname{Hg}\left(X_{0}\right) \leq g+1$. This gives the first assertion of the lemma.

We must now construct a compact connected 3-dimensional submanifold $Y$ of $X_{0}$ such that Properties (1)-(5) hold for $Y$ when $X$ and $S$ are replaced by $X_{0}$ and $S_{0}$.

We have depicted the one-handle $T$ attached to $S$ in figure 5.3, along with some of the objects defined above. We fix a regular neighborhood $R_{1}$ of 
$K \cap S$ in $S$, and observe that $R_{1}$ is a regular neighborhood of $f(D) \cap S=\Gamma$ in $S$. It therefore follows from Lemma 5.9 that $R_{1}$ is a planar surface with three boundary curves. Since $S$ is identified with $\Sigma \times\{1\}$, we have $R_{1}=$ $R \times\{1\}$ for some $R \subset \Sigma$. We set $Y=(R \times I) \cup K \subset(\Sigma \times I) \cup K=X_{0}$.

By construction we have $\overline{X-Y}=(\overline{\Sigma-R}) \times I$ and $(\overline{X-Y}) \cap S_{0}=$ $(\overline{\Sigma-R}) \times\{0\}$. This implies property (5) for $Y$.

To show that $Y$ has property (2), we first observe that $Y=(R \times I) \cup$ $K=(R \times I) \cup T \cup \eta$, and that $T$ and $\eta$ are disjoint from $S_{0}=\Sigma \times\{0\}$ (since their intersection with $\mathcal{C}=\Sigma \times I$ is contained in $S=\Sigma \times\{1\})$. Hence $Y \cap$ $\partial X_{0}$ is the disjoint union of $Z_{0} \doteq R \times\{0\}$ and $Z_{1} \doteq\left(R_{1} \cup T \cup \eta\right) \cap \partial X_{0} \not \subset$ $S_{0}$. The 2-manifold $Z_{0}$ is homeomorphic to $R_{1}$, and is therefore a planar surface with three boundary curves.

To determine the topological type of $Z_{1}$, we first consider the 2-manifold $N \doteq\left(R_{1} \cup T\right) \cap \partial(\mathcal{C} \cup T)$. We may obtain $N$ from $R_{1}$ by removing the interior of $T \cap R_{1}$, which is a union of two disjoint disks, and attaching the annulus $\overline{(\partial T)-\left(T \cap R_{1}\right)}$. Since $R_{1}$ is connected and $\bar{\chi}\left(R_{1}\right)=1$, it follows that $N$ is connected and that $\bar{\chi}(N)=3$. The surface $N$ contains the simple closed curves $f\left(\partial D^{\prime}\right)$ and $\partial \delta$, which by 5.11 .2 meet transversally in exactly three points. In particular, their mod 2 homological intersection number in $N$ is equal to 1 . Hence $f\left(\partial D^{\prime}\right)$ does not separate the connected surface $N$. The 2-manifold $Z_{1}$ is obtained from $N$ by removing the interior of the annulus $N \cap \eta$ and attaching the two components of $\overline{(\partial \eta)-(N \cap \eta)}$, which are disks. Since $\eta$ is a regular neighborhood of $f\left(D^{\prime}\right)$ in $\overline{X-T}$, the annulus $N \cap \eta$ is a regular neighborhood of the nonseparating curve $f\left(\partial D^{\prime}\right)$ in $N$. Hence $Z_{1}$ is connected, and so $Z_{0}$ and $Z_{1}$ are the components of $Y \cap \partial X_{0}$. Furthermore, we have $\partial Z_{1}=\partial R_{1}$, and since $R_{1}$ is a planar surface with three boundary curves, $Z_{1}$ has three boundary curves. Since in addition we have $\bar{\chi}\left(Z_{1}\right)=\bar{\chi}(N)-2=1$, the surface $Z_{1}$ must be planar.

This establishes property (2) for $Y$.

We now turn to the verification of properties (3) and (4). By construction the pair $\left(Y, Z_{0}\right)$ is homotopy equivalent to $\left(R_{1} \cup f(D), R_{1}\right)$. Hence it suffices to show that if $\star \in \operatorname{int} R_{1}$ is a base point, then $\pi_{1}\left(R_{1} \cup f(D), \star\right)$ is free of rank 2 and has an ordered basis $(x, y)$, such that the image of some geometric basis of $\pi_{1}\left(R_{1}, \star\right)$ under the inclusion homomorphism is either $\left(x, y^{2} x y\right)$ or $\left(x, y^{2} x y^{-1}\right)$.

We fix an ordered basis $\left(t, u_{1}, u_{2}\right)$ of the rank-3 free group $\pi_{1}\left(R_{1} \cup \alpha, \star\right)$ having the properties stated in the conclusion of Lemma 5.9. In particular, if $\star \in \operatorname{int} R_{1}$ is any base point, $\pi_{1}\left(R_{1} \cup \alpha, \star\right)$ is free on the generators $t, u_{1}$ and $u_{2}$. Furthermore, $R_{1} \cup f(D)$ is obtained from $R_{1} \cup \alpha$ by attaching a 2-cell, and the attaching map realizes either the conjugacy class of $t^{2} u_{1} t u_{2}$ or that 
of $t^{2} u_{1} t^{-1} u_{2}$ in $\pi_{1}\left(R_{1} \cup \alpha, \star\right)$. Hence $\pi_{1}\left(R_{1} \cup f(D), \star\right)$ is given by either the presentation

$$
\left|t, u_{1}, u_{2}: t^{2} u_{1} t u_{2}=1\right|
$$

in the theta case, or the presentation

$$
\left|t, u_{1}, u_{2}: t^{2} u_{1} t^{-1} u_{2}=1\right|
$$

in the eyeglass case.

Let $\bar{t}$ and $\bar{u}_{i}$ denote the respective images in $\pi_{1}\left(R_{1} \cup f(D), \star\right)$ of the generators $t$ and $u_{i}$ of the free group on $t, u_{1}$ and $u_{2}$. From the properties of the basis $\left(t, u_{1}, u_{2}\right)$ stated in the conclusion of Lemma 5.9, it follows that $u_{1}$ and $u_{2}$ are the images of the elements of a geometric basis of $\pi_{1}\left(R_{1}, \star\right)$ under the inclusion homomorphism $\pi_{1}\left(R_{1}, \star\right) \rightarrow \pi_{1}\left(R_{1} \cup f(D), \star\right)$. On the other hand, it is clear from presentation (5.11.3) or (5.11.4) that $\pi_{1}\left(R_{1} \cup f(D), \star\right)$ is free on the generators $x \doteq \bar{u}_{1}$ and $y \doteq \bar{t}^{-1}$. Furthermore, in the theta case, we have $\bar{u}_{1}=x$ and $\bar{u}_{2}=y x^{-1} y^{2}$; while in the eyeglass case, we have $\bar{u}_{1}=x$ and $\bar{u}_{2}=y^{-1} x^{-1} y^{2}$. Thus properties (3) and (4) of $Y$ are established.

To prove that $Y$ has property (1), we first observe that by construction the frontier of $Y$ is $\partial R \times I$. Hence each component $A$ of the frontier has the form $c \times I$, where $c$ is a component of $\partial R$. Thus $A$ is an annulus having one boundary curve in the component $\Sigma \times\{0\}$ of $\partial X_{0}$. The other boundary curve of $A$ is contained in $\Sigma \times\{1\}$ and is therefore disjoint from $S_{0}=\Sigma \times\{0\}$. In particular, $A$ has its boundary curves in distinct components of $\partial X_{0}$. To prove that $A$ is essential, it therefore suffices to prove that it is $\pi_{1}$-injective in $X_{0}$. This in turn reduces to showing that $A$ is $\pi_{1}$-injective (4.1) in $Y$ and in $\overline{X_{0}-Y}$.

To prove $\pi_{1}$-injectivity of $A$ in $Y$, we observe that since the surface $R$ is homeomorphic to a regular neighborhood of $\Gamma$ in $S$, we have $\bar{\chi}(R)=1$. Hence the component $c$ of $\partial R$ is $\pi_{1}$-injective in $R$. This implies that $c \times\{0\}$ is $\pi_{1}$-injective in $Z_{0}=R \times\{0\}$. Since $Z_{0}$ is $\pi_{1}$-injective in $Y$ by property (4), it follows that $c \times\{0\}$ is $\pi_{1}$-injective in $Y$, and hence that $A$ is $\pi_{1}$-injective in $Y$.

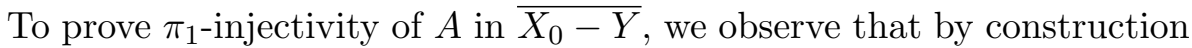
we have $\overline{X_{0}-Y}=\overline{\Sigma-R} \times I \subset(\Sigma \times I) \cup K=X_{0}$. Since $A=c \times I$, it suffices to prove that the boundary component $c$ of $R$ is $\pi_{1}$-injective in $\overline{\Sigma-R}$, or equivalently that no component of $\overline{\Sigma-R}$ is a disk. But this follows immediately from 5.11.1. Thus property (1) of $Y$ is established. 
Remark 5.12. It follows from properties (3) and (4) of $Y$, as stated in the conclusion of Lemma 5.11, that the inclusion homomorphism $H_{1}\left(Z_{0} ; \mathbb{Z}_{2}\right) \rightarrow$ $H_{1}\left(Y ; \mathbb{Z}_{2}\right)$ is an isomorphism.

Lemma 5.13. Suppose that $X$ is a trimonic manifold relative to $S$. Then $\partial X$ has exactly one component $T \neq S$, whose genus is equal to that of $S$, and $T$ is $\pi_{1}$-injective in $X$.

Proof. Let us fix a compact, connected 3-dimensional submanifold $Y$ of $X$ having properties (1)-(5) stated in the conclusion of Lemma 5.11. Define $Z_{0}$ and $Z_{1}$ as in the statement of property (2) of that lemma. According to property (2), we have $Z_{0} \subset S$, and $Z_{1}$ is contained in a component $T \neq S$ of $\partial X$. If $Q$ is a component of $\overline{X-Y}$, then by property (5) of $Y, Q$ is a trivial $I$-bundle. By property (1), each component of the frontier of $Q$ is an essential annulus with one boundary component in $Z_{0}$ and one in $Z_{1}$. Thus the components of $\partial_{h} Q$ may be labeled $F_{0}$ and $F_{1}$ in such a way that $F_{0}$ meets $Z_{0} \subset S$ and $F_{1}$ meets $Z_{1} \subset T$. Note that $F_{0}$ and $F_{1}$ are homeomorphic. Since this holds for each component of $\overline{X-Y}$, and since $Z_{0}$ and $Z_{1}$ are homeomorphic, it follows that $S$ and $T$ have the same Euler characteristic, and furthermore that they are the only components of $\partial X$.

It remains to show that $T$ is $\pi_{1}$-injective in $X$. According to Proposition 4.2 , it suffices to show that the inclusion homomorphism $H_{1}\left(S ; \mathbb{Z}_{2}\right) \rightarrow$ $H_{1}\left(M ; \mathbb{Z}_{2}\right)$ is surjective. For this purpose we set $\mathcal{Q}=\overline{X-Y}$ and $\partial_{0} \mathcal{Q}=$ $\mathcal{Q} \cap S$, we let $F$ denote the frontier of $Y$ in $X$, and we consider the commutative diagram

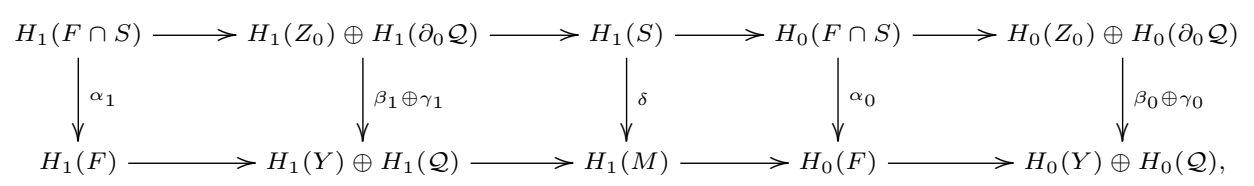

where all homology groups are defined with $\mathbb{Z}_{2}$-coefficients, the rows are segments of Mayer-Vietoris exact sequences, and the homomorphisms $\alpha_{i}$, $\beta_{i}, \gamma_{i}$ and $\delta$ are induced by inclusion.

Since $Z_{0} \subset S$ and $Z_{1} \subset T$, each component of $F$ is an annulus with exactly one boundary curve in $S$. Hence the maps $\alpha_{0}$ and $\alpha_{1}$ are isomorphisms.

If $Q$ is any component of $\mathcal{Q}$, then property (5) of $Y$, as stated in Lemma 5.11 , implies that $Q$ may be given the structure of a trivial $I$-bundle over a 2-manifold in such a way that $Q \cap \partial X$ is the horizontal boundary of $Q$. Since $Q$ must contain at least one component of the frontier of $Y$, exactly 
one component of the horizontal boundary of $Q$ lies in $S$. It follows that $\gamma_{0}$ and $\gamma_{1}$ are isomorphisms. The map $\beta_{0}$ is an isomorphism because $Z_{0}$ and $Y$ are both connected, while $\beta_{1}$ is an isomorphism by Remark 5.12.

Since the $\alpha_{i}, \beta_{i}$ and $\gamma_{i}$ are isomorphisms, it follows from the Five Lemma that $\delta$ is an isomorphism. In particular it is surjective, as required.

Lemma 5.14. Suppose that $X$ is a nondegenerate trimonic manifold relative to a component $S$ of $\partial X$. Then there is no normal book of I-bundles $\mathcal{W}$ with $|\mathcal{W}|=X$.

Proof. Let us fix a compact, connected 3-dimensional submanifold $Y$ of $X$ having properties (1)-(5) of the conclusion of Lemma 5.11. Define $Z_{0}$ as in the statement of property (2) there.

Suppose that $X=|\mathcal{W}|$ for some normal book of $I$-bundles $\mathcal{W}$. Then properties (1) and (2) of $Y$, as stated in 5.11, give hypotheses (4.9) and (4.9) of Lemma 4.9. According to Remark 5.12, the inclusion homomorphism $H_{1}\left(Z_{0} ; \mathbb{Z}_{2}\right) \rightarrow H_{1}\left(Y ; \mathbb{Z}_{2}\right)$ is an isomorphism. This is hypothesis (4.9) of Lemma 4.9 .

Let us fix a base point $\star \in Z_{0}$. According to properties (2)-(4) of $Y$ stated in the conclusion of Lemma $5.11, Z_{0}$ is a planar surface with three boundary curves, and the group $\pi_{1}(Y)$ is free of rank 2, and there exists a pair of generators $(x, y)$ of $\pi_{1}(Y, \star)$, such that either the pair $\left(x, y x^{-1} y^{2}\right)$ or the pair $\left(x, y^{-1} x^{-1} y^{2}\right)$ is the image of some geometric basis of $\pi_{1}\left(Z_{0}, \star\right)$ under the inclusion homomorphism $\pi_{1}\left(Z_{0}, \star\right) \rightarrow \pi_{1}(Y, \star)$. Since neither of the pairs $\left(x, y x^{-1} y^{2}\right)$ or $\left(x, y^{-1} x^{-1} y^{2}\right)$ generates the free group on $x$ and $y$, the inclusion homomorphism $\pi_{1}\left(Z_{0}, \star\right) \rightarrow \pi_{1}(Y, \star)$ is not surjective. Hence Lemma 4.9 implies that there is a solid torus $L \subset Y$ such that $A \doteq L \cap Z_{0}$ is an annulus which is homotopically nontrivial in $|\mathcal{W}|$, and the inclusion homomorphism $H_{1}(A ; \mathbb{Z}) \rightarrow H_{1}(L, \mathbb{Z})$ is not surjective.

Let $c$ denote a core curve of the annulus $A$. Since $c$ is in particular homotopically nontrivial in $Z_{0}$, which is a planar surface with three boundary curves, $c$ is parallel to one of the boundary curves of $Z_{0}$. In view of the definition of a geometric basis, it follows that the conjugacy class in $\pi_{1}(Y, \star)$ defined by a suitably chosen orientation of $c$ is represented by one of the elements $x, y x^{-1} y^{2}, x^{-1} y x^{-1} y^{2}, y^{-1} x^{-1} y^{2}$ or $x^{-1} y^{-1} x^{-1} y^{2}$. On the other hand, since the inclusion homomorphism $H_{1}(A ; \mathbb{Z}) \rightarrow H_{1}(L, \mathbb{Z})$ is not surjective, a representative of a conjugacy class in $\pi_{1}(Y, \star)$ defined by $c$ must be an $n$th power in $\pi_{1}(Y, \star)$ for some $n \neq \pm 1$. But none of the elements $x$, $y x^{-1} y^{2}, x^{-1} y x^{-1} y^{2}, y^{-1} x^{-1} y^{2}$ or $x^{-1} y^{-1} x^{-1} y^{2}$ is a proper power in the free group on $x$ and $y$. This contradiction completes the proof. 


\section{With a $(1,1,1)$ hexagon}

In this section and the next we will be working with hyperbolic 3-manifolds with totally geodesic boundary. As is customary in low-dimensional topology, we shall implicitly carry over the PL results proved in Sections 5 and 4 to the smooth category. For example, to say that a smooth manifold $X$ is a trimonic manifold relative to a component $S$ of $\partial X$ means that the pair $(X, S)$ is topologically homeomorphic to a $\mathrm{PL}$ pair $\left(X^{\prime}, S^{\prime}\right)$ such that $X^{\prime}$ is a trimonic manifold relative to $S^{\prime}$. Likewise, to say that a smooth manifold $X$ has the form $|\mathcal{W}|$ for some normal book of $I$-bundles $\mathcal{W}$ means that $X$ is topologically homeomorphic to a PL manifold which has the form $|\mathcal{W}|$ for some normal book of $I$-bundles $\mathcal{W}$.

Here we will describe some topological consequences of the presence of a $(1,1,1)$ hexagon in $\widetilde{N}$, when the shortest return path of $N$ is not too long. The main result of the section, Proposition 6.8, asserts the existence of a trimonic manifold in $N$ under these circumstances. Below we prove a series of separate lemmas concerning the geometry of $(1,1,1)$ hexagons, from which the proposition follows quickly. The first follows from [17, Lemma 3.2], but for self-containedness we prove it here.

Lemma 6.1. Let $N$ be a compact hyperbolic 3-manifold with totally geodesic boundary, and $\lambda, \lambda^{\prime} \subset \widetilde{N}$ short cuts with length $\ell_{1}$. Then $\lambda=\lambda^{\prime}$ or $\lambda \cap \lambda^{\prime}=\emptyset$.

Proof. Suppose $\lambda$ intersects $\lambda^{\prime}$ at a single point $y \in \lambda$. Let $\Pi$ be the component of $\partial \widetilde{N}$ containing the endpoint $x$ of $\lambda$ closest to $y$, and let $\Pi^{\prime}$ be the component containing the endpoint $x^{\prime}$ of $\lambda^{\prime}$ closest to $y$. The subarcs $[x, y]$ and $\left[x^{\prime}, y\right]$ of $\lambda$ and $\lambda^{\prime}$, respectively, each have length at most $\ell_{1} / 2$. They meet at $y$ at an angle properly less than $\pi$, since $\lambda \neq \lambda^{\prime}$ and each is geodesic. But then $\Pi$ and $\Pi^{\prime}$ are components of $\partial \widetilde{N}$ at distance less than $\ell_{1}$, so they are equal. But since $\lambda$ and $\lambda^{\prime}$ are geodesic arcs, each perpendicular to $\Pi$, in $\widetilde{N}$ they are disjoint or equal, a contradiction.

Remark 6.2. Suppose $N$ is a compact hyperbolic 3-manifold with totally geodesic boundary and $g: \widetilde{N} \rightarrow \widetilde{N}$ is a covering transformation. If $\lambda$ is a short cut with length $\ell_{1}$, then according to Lemma 6.1 , either $g(\lambda)=\lambda$ or $g(\lambda) \cap \lambda=\emptyset$. But in the first case, $g$ would fix a point in $\lambda$, a contradiction. It follows that every short cut of length $\ell_{1}$ is embedded in $N$ by the universal cover. 
Lemma 6.3. Let $N$ be a compact hyperbolic 3-manifold with $\partial N$ connected, totally geodesic and of genus 2 , and suppose $\ell_{1}$ satisfies the bound below:

$$
\cosh \ell_{1}<\frac{\cos (2 \pi / 9)}{2 \cos (2 \pi / 9)-1}=1.4396 \ldots
$$

Then $\ell_{2}>\ell_{1}$; ie, the shortest return path in $N$ is unique.

Proof. Suppose $\ell_{2}=\ell_{1}$. Applying the right-angled hexagon rule as in the proof of Lemma 2.6, we find that each of $d_{11}, d_{12}$, and $d_{22}$ is at least $2 R$, for $R$ the function of $\ell_{1}$ defined there. Then a disk of radius $R$ is embedded around each of the feet of $\lambda_{1}$ and $\lambda_{2}$, so that none of these disks overlap. Boröczky's bound on the radius of four disks of equal area embedded without overlapping on a surface of genus 2 is the quantity $R^{\prime \prime}$ defined in Lemma 2.9. Setting $R=R^{\prime \prime}$ and solving for $\ell_{1}$ yields the quantity of the bound above.

Lemma 6.4. Let $N$ be a compact hyperbolic 3-manifold with totally geodesic boundary. Suppose $C$ and $C^{\prime}$ are distinct $(1,1,1)$ hexagons in $\widetilde{N}$ with exterior edges on the same component of $\partial \widetilde{N}$. Then $C \cap C^{\prime}$ is either empty or a single interior edge.

Proof. Let $e$ and $e^{\prime}$ be exterior edges of $C$ and $C^{\prime}$ on the same component of $\partial \widetilde{N}$. The endpoints of $e$ and $e^{\prime}$ are feet of lifts of the shortest return path; any such pair has distance at least $d_{11}$. Since $C$ and $C^{\prime}$ are distinct, so are $e$ and $e^{\prime}$; if they share an endpoint then $C \cap C^{\prime}$ consists of an interior edge.

Otherwise, $e$ is a geodesic arc of length $d_{11}$ connecting its endpoints $a$ and $b$ and $e^{\prime}$ an arc of the same length connecting its endpoints $a^{\prime}$ and $b^{\prime}$, with $d\left(a, a^{\prime}\right), d\left(a, b^{\prime}\right), d\left(b, a^{\prime}\right)$ and $d\left(b, b^{\prime}\right)$ all at least $d_{11}$. Some hyperbolic trigonometry shows that any point at distance at least $d_{11}$ from $a$ and $b$ satisfies $\cosh \ell \geq \cosh d_{11} / \cosh \left(d_{11} / 2\right)$, where $\ell$ is its distance from $e$. Twice this distance is larger than $d_{11}$; thus $e^{\prime}$ does not cross $e$, and so $C \cap C^{\prime}=\emptyset$.

Remark 6.5. Suppose $N$ is a compact hyperbolic 3-manifold with totally geodesic boundary and $g: \widetilde{N} \rightarrow \widetilde{N}$ is a covering transformation. If $C$ is a $(1,1,1)$ hexagon, and an external edge of $g(C)$ intersects an external edge of $C$, then by Lemma 6.4, either $g(C)=C$ or $g(C) \cap C$ is a single internal edge. In the former case, $g$ fixes a point in $C$, a contradiction. It follows that the union of the interiors of external edges of $C$ projects homeomorphically to $N$. 
Lemma 6.6. Let $N$ be a compact hyperbolic 3-manifold with totally geodesic boundary, and suppose cosh $\ell_{1} \leq 1.215$. If $C$ is a $(1,1,1)$ hexagon in $\widetilde{N}$ and $\lambda$ is a short cut of length $\ell_{1}$, then $\lambda$ is an internal edge of $C$ or $\lambda \cap C=\emptyset$.

Proof. Suppose $\lambda$ and $C$ are as in the lemma, and $\lambda \cap C$ is nonempty. Let $\Pi_{1}$ and $\Pi_{2}$ be the components of $\partial \widetilde{N}$ containing the endpoints of $\lambda$, and let $\Pi$ be the geodesic plane containing $C$. Suppose first that $\lambda \subset \Pi$. If $\lambda$ is not contained in $C$, let $x$ be an endpoint of $\lambda \cap C$ contained in the interior of $\lambda$. Then $x$ is a point of intersection between $\lambda$ and an internal edge of $C$, since the external edges of $C$ are contained in $\partial \widetilde{N}$ and $\lambda$ is properly embedded. But each internal edge of $C$ is a short cut with length $\ell_{1}$, so this contradicts Lemma 6.1. It follows that if $\lambda \subset \Pi, \lambda \subset C$. But $C$ intersects only three components of $\partial \widetilde{N}$ - the components joined by its internal edges. Hence since $C$ intersects $\Pi_{1}$ and $\Pi_{2}, \lambda$ is an internal edge of $C$.

Now suppose $\lambda$ is not contained in $\Pi$. Then $\lambda$ intersects $C$ transversely in a single point $x$. There is a component $\Pi^{\prime}$ of $\partial \widetilde{N}$ such that $\Pi^{\prime} \cap \Pi$ contains an external edge of $C$ and $x$ is distance at most $A$ from $\Pi^{\prime}$. (Recall that $A$, defined in (3.1.1), is a side length of a fundamental domain for the symmetry group of $C$; see figure 6.1.) Then the distance from $\Pi^{\prime}$ to each of $\Pi_{1}$ and $\Pi_{2}$ is less than $A+\ell_{1}$. By Lemma 3.8, $\ell_{2}$ is at least twice $A$ and $\ell_{1}$; hence the distance from $\Pi^{\prime}$ to each of $\Pi_{1}$ and $\Pi_{2}$ is $\ell_{1}$, since it is less than $\ell_{2}$. By Lemma 2.3, there is a $(1,1,1)$ hexagon $C^{\prime}$ with $\lambda$ as an internal edge and external edges in $\Pi^{\prime}, \Pi_{1}$, and $\Pi_{2}$. But then $C^{\prime} \cap C$ contains $\lambda \cap C$, contradicting Lemma 6.4.

Lemma 6.7. Let $N$ be a compact hyperbolic 3-manifold with totally geodesic boundary, and suppose $\cosh \ell_{1} \leq 1.215$. If $C$ and $C^{\prime}$ are distinct $(1,1,1)$ hexagons in $\widetilde{N}$, then $C \cap C^{\prime}$ is empty or a single internal edge of each.

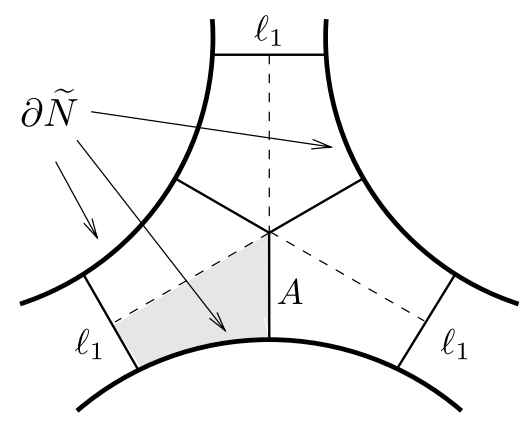

Figure 6.1: A $(1,1,1)$ hexagon in $\widetilde{N}$, with a fundamental domain for its symmetry group shaded. 
Proof. Suppose $C$ and $C^{\prime}$ are distinct $(1,1,1)$ hexagons in $\tilde{N}$, and $C \cap C^{\prime} \neq$ $\emptyset$. By Lemma 6.4, the lemma holds if there is a component of $\partial \widetilde{N}$ containing external edges of both $C$ and $C^{\prime}$, thus we may assume that this is not the case. It follows that no external edge of $C$ contains a point of $C \cap C^{\prime}$ and vice versa, since by Lemma $2.4, C \cap \partial \widetilde{N}$ is precisely the union of its external edges. Let $\Pi$ be the geodesic hyperplane containing $C$. If $C^{\prime} \subset \Pi$, then $C \cap C^{\prime}$ is a two-dimensional subpolyhedron of $C$, and each vertex of $C \cap C^{\prime}$ is an intersection point between internal edges, by the above. But these are all short cuts of length $\ell_{1}$, contradicting Lemma 6.1.

If $C^{\prime}$ is not contained in $\Pi$, it intersects $C$ transversely in a geodesic arc, whose endpoints are points of intersection of internal edges of one with the other. But such intersections violate Lemma 6.6, since the internal edges of each are short cuts of length $\ell_{1}$, a contradiction.

Proposition 6.8. Let $N$ be a compact, orientable hyperbolic 3-manifold with $\partial N$ connected, totally geodesic and of genus 2 . Suppose that $\cosh \ell_{1} \leq$ 1.215 , and there is a $(1,1,1)$ hexagon in $\tilde{N}$. There is a submanifold $X \subset N$ with $\partial N \subset X$, such that $X$ is a trimonic manifold relative to $\partial N$.

Proof. Let $N$ be as in the hypotheses, and fix a $(1,1,1)$ hexagon $C \subset \tilde{N}$. Let $f: C \rightarrow N$ be the restriction of the universal covering, and let $X$ be a regular neighborhood of $\partial N \cup f(C)$. Since $\cosh \ell_{1} \leq 1.215$, Lemma 6.3 implies that $N$ has a unique shortest return path $\alpha$, hence each internal edge of $C$ projects to $\alpha$ by $f$. The preimage of $\alpha$ is a union of short cuts with length $\ell_{1}$, hence Lemma 6.6 implies property (1) of Definition 5.7. Remark 6.2 now immediately implies property (2) of the definition. property (3) follows from Remark 6.5 and Lemma 6.7. Properties (4) and (5) follow from Lemma 2.4, and (6) holds by construction.

Proposition 6.9. Let $N$ be a compact, orientable hyperbolic 3-manifold with $\partial N$ connected, totally geodesic and of genus 2 , such that $\cosh \ell_{1} \leq 1.215$ and there is a $(1,1,1)$ hexagon in $\tilde{N}$. The trimonic submanifold $X \subset N$ supplied by Proposition 6.8 is nondegenerate.

Proof. Let $N$ satisfy the hypotheses, and as in the proof of Proposition 6.8 let $C \subset \widetilde{N}$ be a $(1,1,1)$ hexagon, $f: C \rightarrow N$ the restriction of the universal cover, and $\alpha \subset N$ the shortest return path. Below we will borrow wholesale the constructions and notation from the proof of Lemma 5.9, with $C$ here in the role of $D$ there and $\partial N$ in the role of $S$.

Recall that the internal edges $a_{i}$ and external edges $b_{i}$ of $C, i \in\{0,1,2\}$, are enumerated so that $a_{i}$ shares a vertex with $b_{i}$ and $b_{i-1}$ for each $i$, and $\partial C$ 
is oriented so that $\phi_{0} \doteq f \mid a_{0}$ induces the same orientation on $\alpha$ as $\phi_{1} \doteq f \mid a_{1}$. Then $\alpha$ is given the orientation induced by $\phi_{0}$ and $\phi_{1}$, with initial and terminal vertices $V$ and $W$, respectively. For each $i \in\{0,1,2\}$, the edge $\beta_{i}=f\left(b_{i}\right)$ of $\Gamma=\Gamma_{f}$ is given the induced orientation from $b_{i}$.

Cases I and II in the proof of Lemma 5.9 are distinguished according to whether $\phi_{2} \doteq f \mid a_{2}$ is orientation preserving or reversing, respectively. In Case I, $\Gamma=\overline{\beta_{0} \cup \beta_{1} \cup \beta_{2}}$ is a theta graph, and in Case II it is an eyeglass. (Recall that $\beta_{i}=f\left(\right.$ int $\left.b_{i}\right), i \in\{0,1,2\}$.)

For each $i \in\{0,1,2\}$, let $\Pi_{i}$ be the component of $\partial \tilde{N}$ containing $b_{i}$. Suppose that $U$ is a component of $\partial N-\Gamma$ which is homeomorphic to an open disk, and let $U_{0} \subset \Pi_{0}$ be a component of the preimage of $U$ under the universal covering map. Then $U_{0}$ is projected homeomorphically to $U$, and $\bar{U}_{0}$ is a compact polygon in $\Pi_{0}$ with edges projecting to the $\beta_{i}$, hence covering translates of the $b_{i}$. Since the $b_{i}$ are geodesic arcs, $\bar{U}_{0}$ has at least three edges. Since each vertex $v$ of $\bar{U}_{0}$ projects to $V$ or $W$, and $U_{0}$ projects homeomorphically, $\bar{U}_{0}$ has at most six vertices. Hence $\bar{U}_{0}$ is an $n$-gon for $n$ between 3 and 6 .

Suppose edges $b$ and $b^{\prime}$ incident to a single vertex $v$ of $\bar{U}_{0}$ were identified in $\Gamma$. The covering transformation $f$ taking $b$ to $b^{\prime}$ is orientation preserving on $\tilde{N}$, so it preserves the boundary orientation on $\Pi_{0}$. Give $U_{0}$ this orientation, and orient $b$ and $b^{\prime}$ as arcs in the boundary of $U_{0}$. Since $f\left(U_{0}\right)$ does not intersect $U_{0}, f\left(\bar{U}_{0}\right) \cap \bar{U}_{0}=b^{\prime}=f(b)$. Since $f(b)$ has the boundary orientation from $f\left(U_{0}\right)$, its orientation is opposite that of $b^{\prime}$. But then since $v$ is the initial vertex of (say) $b$ and the terminal vertex of $b^{\prime}, f(v)=v$, a contradiction. Hence:

6.9.1. If $v$ is a vertex of $\bar{U}_{0}$, the edges incident to $v$ project to distinct edges of $\Gamma$.

Now suppose $\bar{U}_{0}$ is a triangle. Then two of its vertices are identified in $N$, since $\Gamma$ has only two vertices. The edge joining these vertices projects to an edge joining $V$ to $V$ or $W$ to $W$, so in this case $\Gamma$ is an eyeglass graph. On the other hand, the final vertex of $\bar{U}_{0}$ is not identified with the other two by 6.9 .1 , since an eyeglass graph has only one edge joining each vertex to itself. But then the two edges emanating from the final vertex yield distinct edges of $\Gamma$, joining $V$ to $W$, which does not occur in an eyeglass graph. This is a contradiction.

When $\bar{U}_{0}$ is a pentagon, three of its vertices are identified to $V$ (say) in $\Gamma$. Thus two of these are adjacent in $\bar{U}_{0}$. The edge joining the adjacent vertices of $\bar{U}_{0}$ identified to $V$ joins it to itself in $\Gamma$, hence $\Gamma$ is an eyeglass graph. The 
third vertex identified to $V$ is not adjacent to either of the others, by 6.9.1, since $\Gamma$ has only one edge joining $V$ to itself. Then the edges adjacent to this vertex project to distinct $\beta_{i}$ joining $V$ to $W$, a contradiction.

To rule out the possibility that $\bar{U}_{0}$ is a quadrilateral or hexagon requires counting angles. Recall that $S=\partial N$ is oriented so that $\mathcal{T}_{V}$ is a positive oriented triod (see 5.1). Here $\mathcal{T}_{V}=\left(\sigma_{0}, \sigma_{1}, \sigma_{2}\right)$ in Case I and $\mathcal{T}_{V}=\left(\sigma_{0}, \sigma_{1}, \rho_{2}\right)$ in Case II, where for each $i \in\{0,1,2\}, \rho_{i}$ is the closure of an initial segment of $\beta_{i}$ and $\sigma_{i}$ is the closure of a terminal segment of $\beta_{i-1}$. Define $\theta_{1}$ to be the angle measure from $\sigma_{0}$ to $\sigma_{1}$ at $V$, in the direction prescribed by the orientation on $\partial N$. In Case I, we take $\theta_{2}$ to be the angle from $\sigma_{0}$ to $\sigma_{2}$ in the orientation direction, and in Case II we let $\theta_{2}$ be the angle from $\sigma_{0}$ to $\rho_{2}$. Then $0<\theta_{1}<\theta_{2}<2 \pi$.

Recall that $C$ is a totally geodesic hexagon in $\tilde{N}$ by Lemma 2.3, and the covering projection $f$ immerses $C$ in $N$ isometrically. Then appealing to figure 5.2, we note that $\theta_{1}$ is the angle from $\rho_{1}$ to $\rho_{0}$ at $W$, measured in the orientation direction. This is because the homeomorphism $\psi: \delta_{V} \rightarrow \delta_{W}$ defined in the proof of Lemma 5.9 is orientation reversing. Similarly, in Case I $\theta_{2}$ is the the angle from $\rho_{2}$ to $\rho_{0}$ at $W$ in the orientation direction, and in Case II it is the angle from $\sigma_{2}$ to $\rho_{0}$.

If $\bar{U}_{0}$ is a quadrilateral, then two of its edges are sent by $f$ to the same edge of $\Gamma$. These are opposite, by 6.9.1; hence the image in $\Gamma$ of each of the remaining edges joins a vertex to itself. Then $\Gamma$ is an eyeglass graph, the pair of opposite edges identified by $f$ project to $\beta_{0}$, and the other two edges project to $\beta_{1}$ and $\beta_{2}$. Abusing notation slightly, we label the edges projecting to $\beta_{0}$ by $b_{0}$ and $b_{0}^{\prime}$, and similarly label the edge projecting to $\beta_{i}$ by $b_{i}, i=1,2$. (These are covering translates of the corresponding edges of $C$.) We give each edge of $\bar{U}_{0}$ an orientation matching that of its correspondent in $\Gamma$.

Orient $U_{0}$ so that $f \mid U_{0}$ preserves orientation. We may assume, by switching the labels of $b_{0}$ and $b_{0}^{\prime}$ and/or replacing $C$ with a covering translate if necessary, that the prescribed orientation on $b_{0} \subset C$ matches the boundary orientation which it inherits from $\bar{U}_{0}$. The terminal endpoint of $b_{0}$ is sent to $V$, and a terminal segment is sent to the interior of $\sigma_{1}$ by definition. Since the orientation on $\partial N$ is chosen so that $\left(\sigma_{0}, \sigma_{1}, \rho_{2}\right)$ is a positively oriented triod, the orientation $\sigma_{1}$ inherits from $\beta_{0}$ matches the boundary orientation from the component of $\delta_{V}-\left(\sigma_{0} \cup \sigma_{1} \cup \sigma_{2}\right)$ bounded by it and $\rho_{2}$. It follows that the terminal endpoint of $b_{0}$ is the initial endpoint of $b_{2}$, and the dihedral angle of $\bar{U}_{0}$ at this vertex is $\theta_{2}-\theta_{1}$.

Since the orientation on $b_{0}^{\prime}$ is opposite that induced by $\bar{U}_{0}$, the dihedral angle of $\bar{U}_{0}$ at the common terminal endpoint of $b_{0}^{\prime}$ and $b_{2}$ is $\theta_{1}$, the dihedral angle in $\delta_{V}$ between $\sigma_{0}$ and $\sigma_{1}$. Arguing as above, we find that the dihedral 
angle of $\bar{U}_{0}$ at the initial endpoint of $b_{0}$, which is the terminal endpoint of $b_{1}$, is $2 \pi-\theta_{2}$. (Recall that the homeomorphism $\psi: \delta_{V} \rightarrow \delta_{W}$ visible in figure 5.2 as projection upward is orientation reversing.) The dihedral angle at the final vertex is then $\theta_{1}$. Thus the sum of the dihedral angles is $2 \pi+\theta_{1}$, contradicting the well known fact that the dihedral angle sum of a hyperbolic quadrilateral is less than $2 \pi$.

Now suppose $\bar{U}_{0}$ is a hexagon. Then the projection of $\bar{U}_{0}$ contains each of $\delta_{V}$ and $\delta_{W}$, since $\bar{U}_{0}$ has six vertices and each of $V$ and $W$ has valence 3. Thus the sum of the dihedral angles around vertices of $\bar{U}_{0}$ is $4 \pi$. But a hyperbolic hexagon has dihedral angle sum less than $4 \pi$, a contradiction. It follows that no component of $\partial N-\Gamma$ is homeomorphic to an open disk. Since $f: C \rightarrow N$ is a defining hexagon (in the sense of Definition 5.10) for the submanifold $X$ defined in Proposition 6.8, and since $\Gamma=\Gamma_{f}$, the trimonic manifold $X$ is nondegenerate.

\section{Putting it all together}

In this section we prove the theorems stated in the introduction. Here we make much use of terminology and results from [6]. Of particular importance is the term " $(g, h)$-small," from Definition 1.2 there:

Definition 7.1. Let $g$ be an integer $\geq 2$, let $h$ be a positive real number, and let $M$ be an orientable, irreducible 3-manifold. We shall say that $M$ is $(g, h)$-small if every connected closed incompressible surface in $M$ has genus at least $h$ and every separating connected closed incompressible surface in $M$ has genus at least $g$.

Lemma 7.2. Let $\mathcal{W}$ be a normal book of I-bundles, set $W=|\mathcal{W}|$, and suppose that $\partial W$ is connected and has genus 2 . Then $\operatorname{Hg}(W)=3$.

Proof. Since $\bar{\chi}(W)=\frac{1}{2} \bar{\chi}(\partial W)=1$, there is a unique page $P$ of $\mathcal{W}$ which has negative Euler characteristic; and furthermore, $\bar{\chi}(P)=1$. Since $P \cap \partial W=$ $\partial_{h} P$ is a $\pi_{1}$-injective subsurface of $\partial W$ with Euler characteristic -2 , its complement in $\partial W$ is a disjoint union of annuli. Thus if $B$ is a component of $\overline{W-P}$, then $\partial B$ is a union of annuli in $\overline{\partial W-P}$ and vertical annuli in the frontier of $P$, and is therefore a torus. Since the frontier of $B$ in $W$ consists of essential annuli, $B$ is $\pi_{1}$-injective in $W$, and since $W$ is simple, $B$ is $(2,2)$-small. It now follows from [6, Proposition 2.3] that each component of $\overline{W-P}$ is a solid torus. 
By definition $P$ is an $I$-bundle over a surface $T$. We let $p: P \rightarrow T$ denote the bundle map. If $C$ is a closed disk contained in the interior of $T$, then $\mathcal{H}=p^{-1}(C)$ is a 1-handle in $P$ joining $\partial_{h} P \subset \partial W$ to itself. Let $\delta_{0}$ be an arc embedded in $\overline{T-C}$, so that $\partial \delta_{0}=\delta_{0} \cap \partial(\overline{T-C}) \subset \partial C$ and no arc of $\partial C$ bounds a disk embedded in $\overline{T-C}$ together with $\delta_{0}$. The existence of such an arc can be established using the fact that $\chi(\overline{T-C})=-2$ and standard Morse theory arguments. If $D_{0}$ is a regular neighborhood of $\delta_{0}$ in $\overline{T-C}$, then $\overline{T-C-D_{0}}$ is a possibly disconnected surface with Euler characteristic -1 and no component which is a disk. Let $T_{0}$ be the component with $\chi\left(T_{0}\right)=$ -1 , and let $\beta$ be a component of $\partial T_{0}$ containing an arc of the frontier in $\overline{T-C}$ of $D_{0}$. There is an arc $\delta_{1}$ embedded in $T_{0}$, so that $\partial \delta_{1}=\delta_{1} \cap \partial T_{0} \subset \beta$ and no arc of $\beta$ bounds a disk in $T_{0}$ together with $\delta_{1}$. This follows as above, and we may further assume, after sliding $\partial \delta_{1}$ along $\beta$ if necessary, that $\partial \delta_{1}$ does not intersect the frontier of $D_{0}$. Let $D_{1}$ be a regular neighborhood in $T_{0}$ of $\delta_{1}$ which does not intersect $D_{0}$, and let $D=D_{0} \sqcup D_{1} \subset \overline{T-C}$.

By construction, $\overline{T-C-D}$ is a disjoint union of surfaces with Euler characteristic 0; that is, annuli and Möbius bands. Since $T$ is connected, each component of $\overline{T-C-D}$ has at least one component of its boundary containing arcs of the frontier of $D$. Thus if $\alpha$ is a component of $\partial T$, the component $T^{\prime}$ of $\overline{T-C-D}$ containing $\alpha$ is an annulus, and $\alpha$ is the unique component of $\partial T^{\prime}$ contained in $\partial T$. For $i=0$ or 1 , let $\mathcal{D}_{i}=p^{-1}\left(D_{i}\right) \subset P$, and let $\mathcal{D}=\mathcal{D}_{0} \sqcup \mathcal{D}_{1}$. Each of $\mathcal{D}_{0}$ and $\mathcal{D}_{1}$ is an $I$-bundle over a disk, hence a ball, and each component of $\overline{P-\mathcal{H}-\mathcal{D}}$ is an $I$-bundle over an annulus or Möbius band, hence is a solid torus.

Let $B$ be a component of $\overline{W-P}$. Then the component of $\overline{W-\mathcal{H}-\mathcal{D}}$ containing $B$ is the union of $B$ with a collection of solid torus components of $\overline{P-\mathcal{H}-\mathcal{D}}$. If $B_{1}$ is such a component, by the above $B_{1}$ is an $I$-bundle over an annulus component of $\overline{T-C-D}$ with a unique boundary component $\alpha \subset \partial T$. Let $A=p^{-1}(\alpha)=B \cap B_{1}$, a vertical annulus in the frontier of $P$. Since $A$ is a degree one annulus in $\partial B_{1}$ it follows that $B \cup B_{1}$ is a solid torus. It follows that each component of $\overline{W-\mathcal{H}-\mathcal{D}}$ is a solid torus, so $\overline{W-\mathcal{H}}$ is obtained from a collection of solid tori by adding $\mathcal{D}_{0}$ and $\mathcal{D}_{1}$.

Let $\mathcal{N}_{0}$ be a regular neighborhood of $\partial W$ such that $\mathcal{N}_{0} \cap P$ is a regular neighborhood of $\partial_{h} P$ in $T$ with horizontal frontier. Then $\mathcal{N}_{0} \cap \mathcal{H}$ is a disjoint union of two solid cylinders. Let $V_{0}=\mathcal{N}_{0} \cup \mathcal{H}$. $V_{0}$ is a compression body in $W$ with frontier a surface $S$ of genus 3 . Our description above shows that $V_{1}=\overline{W-V_{0}}$ is the union of a collection of solid tori with $\mathcal{D}_{0} \cap V_{1}$ and $\mathcal{D}_{1} \cap V_{1}$. Each of these has the structure of a 1-handle, since it is a ball and its intersection with its complement in $V_{1}$ consists of two disks. Hence $V_{1}$ is a handlebody and $S$ is a Heegaard surface for $W$. 
Lemma 7.3. Let $N$ be a compact, irreducible, orientable 3-manifold such that $\partial N$ is a connected nonempty surface of genus $>1$. Suppose that $N$ contains no incompressible torus. Let $Y$ be a connected, 3-dimensional submanifold of $N$ such that $\partial N \subset Y$, and such that every component of the frontier of $Y$ in $N$ is a torus. Then $\operatorname{Hg}(N) \leq \mathrm{Hg}(Y)$.

Proof. We argue by induction on the number $m \geq 0$ of components of the frontier of $Y$ in $N$. If $m=0$ we have $N=Y$ and the assertion is trivial. Now suppose that $m>0$, and that for every connected, 3-dimensional submanifold $Z \supset \partial N$ of $N$ whose frontier consists of strictly fewer than $m$ tori, we have $\operatorname{Hg}(N) \leq \operatorname{Hg}(Z)$. Let $T$ denote the frontier of $Y$. Since $T \neq \emptyset$ and each component of $T$ is a torus, the hypothesis implies that $T$ is compressible in $N$. Hence there is a disk $D \subset N$ with $D \cap T=\partial D$, such that $\partial D$ is homotopically nontrivial in the component $T_{0}$ of $T$ containing it. Since $T$ is the frontier of $Y$ in $N$, we must have either $D \cap Y=\partial D$ or $D \subset Y$.

In the case where $D \cap Y=\partial D$, we let $E$ denote a regular neighborhood of $D$ in $\overline{N-Y}$. The component of $\partial(Y \cup E)$ which meets $T_{0}$ is a sphere, which by irreducibility bounds a ball $B \subset N$. Since $\partial N \subset Y$ we cannot have $B \supset Y \cup E$; hence we must have $B \cap(Y \cup E)=\partial B$. It follows that $J \doteq$ $B \cup E$ is a solid torus with $J \cap Y=\partial J=T_{0}$. A Heegaard surface for $Y$ is also a Heegaard surface for $Y \cup J$ (cf. [6, Lemma 2.1]), so that $\operatorname{Hg}(Y \cup$ $J) \leq \mathrm{Hg}(Y)$; but the induction hypothesis implies that $\operatorname{Hg}(N) \leq \mathrm{Hg}(Y \cup J)$. Hence $\operatorname{Hg}(N) \leq \mathrm{Hg}(Y)$.

In the case where $D \subset Y$, we let $E$ denote a regular neighborhood of $D$ in $Y$. The component of the boundary of $\bar{Y}-E$ which meets $T_{0}$ is a sphere, and is isotopic by a small ambient isotopy of $N$ to a sphere $S \subset \operatorname{int} Y$ which separates $T_{0}$ from $\partial N$ in $Y$. Given a Heegaard surface $F$ for $Y$, Haken's lemma (cf. [5, Lemma 1.1]) asserts that there is a 2-manifold $S^{\prime}$ obtained from $S$ by 1-surgeries and isotopies such that each component of $S^{\prime}$ meets $F$ transversally in a single simple closed curve. We will take $F$ to be a minimal-genus Heegaard surface for $Y$. Since $S$ separates $T_{0}$ from $\partial N$, some component $S_{0}$ of $S^{\prime}$ must separate $T_{0}$ from $\partial N$. Let $C$ denote the simple closed curve $S_{0} \cap F$.

Since $N$ is irreducible, $S_{0}$ bounds a ball $B_{0} \subset \operatorname{int} N$. We have $T_{0} \subset \operatorname{int} B_{0}$. Hence the number $k$ of components of $T$ contained in $B_{0}$ is strictly positive. If we set $Z=Y \cup B_{0}$ then $Y \supset \partial N$, and the frontier of $Z$ consists of $m^{\prime}$ tori, where $m^{\prime}=m-k<m$. Hence the induction hypothesis implies that $\mathrm{Hg}(N) \leq \mathrm{Hg}(Z)$. We will complete the proof in this case by showing that $\operatorname{Hg}(Z) \leq \operatorname{Hg}(Y)$. 
Let $H_{1}$ and $H_{2}$ denote the closures of the components of $Y-F$. Then $D_{i}=S_{0} \cap H_{i}$ is a disk with $\partial D_{i}=C$ for $i=1,2$. Set $Z^{\prime}=\overline{Z-B_{0}}=$ $\overline{Y-\left(Y \cap B_{0}\right)}$, for $i=1,2$ set $H_{i}^{\prime}=H_{i} \cap Z^{\prime}$, and let $F^{\prime}=F \cap Z^{\prime}=H_{1}^{\prime} \cap H_{2}^{\prime}$. Then $H_{i}^{\prime}$ is a submanifold of $H_{i}$ whose frontier is $D_{i}$. But $H_{i}$ is a compression body with $\partial_{+} H_{i}=F$, and $D_{i}$ is a properly embedded disk in $H_{i}$ whose boundary lies in $F$. Hence $H_{i}^{\prime}$ is a compression body with $\partial_{+} H_{i}^{\prime}=F^{\prime} \cup D_{i}$ (cf. [3, Corollary B.3]), and genus $\left(F^{\prime}\right) \leq \operatorname{genus}(F)=\mathrm{Hg}(Y)$.

If we choose a properly embedded disk $\Delta$ in $B_{0}$ with $\partial \Delta=C$, we may write $B_{0}=B_{1} \cup B_{2}$, where $B_{i}$ is a ball containing $D_{i}$ for $i=1,2$, and $B_{1} \cap B_{2}=\Delta$. It now follows that $H_{i}^{*} \cdot H_{i}^{\prime} \cup B_{i}$ is a compression body with $\partial_{+} H_{i}^{*}=F^{*} \doteq F^{\prime} \cup \Delta$, and $Z=H_{1}^{*} \cup H_{2}^{*}$. Hence $F^{*}$ is a Heegaard surface for $Z$, and therefore $\operatorname{Hg}(Z) \leq \operatorname{genus}\left(F^{*}\right)=\operatorname{genus}\left(F^{\prime}\right) \leq \mathrm{Hg}(Y)$, as required.

Theorem 7.4. Let $N$ be a compact, orientable hyperbolic 3-manifold with $\partial N$ a connected totally geodesic surface of genus 2 . If $\cosh \ell_{1} \leq 1.215$ and there is a $(1,1,1)$ hexagon in $\widetilde{N}$, then $\operatorname{Hg}(N) \leq 4$ or $\operatorname{vol}(N)>7.32$.

Proof. Let $N$ satisfy the hypotheses of the theorem, and let $X \subset N$ be the codimension-0 submanifold supplied by Proposition 6.8, which is a trimonic manifold with respect to $\partial N$, nondegenerate by Proposition 6.9. Let $T$ be the frontier of $X$ in $N$. By Lemma 5.13, $T$ is a surface of genus 2 which is $\pi_{1}$-injective in $X$.

Let $V=\overline{N-X}$. Then $V$ is a compact, connected, irreducible 3-dimensional submanifold of $N$ with $\partial V=T$. Therefore $\bar{\chi}(V)=1$. By Lemma 5.11, $X$ has Heegaard genus equal to 3 .

We first consider the case in which $V=|\mathcal{W}|$ for some normal book of $I$-bundles $\mathcal{W}$. By Lemma 7.2, we have $\operatorname{Hg}(V)=3$. Amalgamating the Heegaard splittings of $V$ and $X$, each of genus 3, across $T$ yields a Heegaard splitting of $N$ with genus 4 (cf. [20, Remark 2.7], and the definition above it).

Next we consider the case in which $V$ is boundary-irreducible and $V$ does not have the form $|\mathcal{W}|$ for any normal book of $I$-bundles $\mathcal{W}$. Since $V$ is boundary-irreducible and $T$ is $\pi_{1}$-injective in $X$, the surface $T$ is incompressible in $N$. Hence $V$ and $X$ are simple. By Lemma 5.14, $X$ is not homeomorphic to $|\mathcal{W}|$ for any book of $I$-bundles $\mathcal{W}$. Since $V$ is also not homeomorphic to $|\mathcal{W}|$ for any book of $I$-bundles $\mathcal{W}$, it follows from Proposition 4.7 that $\chi\left(\overline{X-\Sigma_{X}}\right)<0$ and $\chi\left(\overline{V-\Sigma_{V}}\right)<0$, where $\Sigma_{V}$ and $\Sigma_{X}$ denote the characteristic submanifolds of $V$ and $X$ relative to their boundaries. According to [6, Definition 1.1], $\operatorname{kish}(V)($ or $\operatorname{kish}(X))$ is the union of all components of $\overline{V-\Sigma_{V}}$ (or respectively $\overline{X-\Sigma_{X}}$ ) having negative Euler characteristic. We therefore have kish $V \neq \emptyset$ and kish $X \neq \emptyset$, so that $\bar{\chi}\left(\overline{X-\Sigma_{X}}\right) \geq 1$ and 
$\bar{\chi}\left(\overline{V-\Sigma_{V}}\right) \geq 1$. Hence $\bar{\chi}(\operatorname{kish} N \backslash \backslash T)=\bar{\chi}(\operatorname{kish}(X))+\bar{\chi}(\operatorname{kish}(V)) \geq 2$, and by Agol et al. [2, Theorem 9.1], the volume of $N$ is greater than 7.32.

There remains the case in which $V$ is boundary-reducible. Let $D$ be an essential properly embedded disk in $V$, and let $E$ denote a regular neighborhood of $D$ relative to $V$. Set $W=\overline{V-E}$ and $Y=X \cup E$. Then $N=Y \cup W$ and $Y \cap W=\partial W$. Furthermore, $\partial W$ has at most two components, and each component of $\partial W$ is a torus. A genus-3 Heegaard surface $F$ for $X$ is also a Heegaard surface for $Y$ (cf. [6, Lemma 2.1]), and so $\operatorname{Hg}(Y) \leq 3$. On the other hand, since $N$ is a compact hyperbolic 3 -manifold with totally geodesic boundary, it is irreducible and contains no incompressible torus. It therefore follows from Lemma 7.3 that $\mathrm{Hg}(N) \leq \mathrm{Hg}(Y) \leq 3$.

Theorem 1.1. Let $N$ be a compact, orientable hyperbolic 3-manifold with $\partial N$ a connected totally geodesic surface of genus 2 . If $\operatorname{Hg}(N) \geq 5$, then $N$ has volume greater than 6.89 .

Proof. Let $N$ satisfy the hypotheses of the theorem, and let $\ell_{1}$ be the length of the shortest return path of $N$. If $\cosh \ell_{1} \geq 1.215$, then by Proposition $3.7, N$ has volume greater than 6.89 . If $\widetilde{N}$ contains no $(1,1,1)$ hexagon, then by Proposition 3.9, $\ell_{1} \geq 1.215$, and Proposition 3.7 again gives the desired volume bound. We thus suppose that $N$ has a $(1,1,1)$ hexagon and $\cosh \ell_{1}<1.215$. But in this case Theorem 7.4 gives a better volume bound of 7.32 , since by hypothesis $\operatorname{Hg}(N) \geq 5$.

Theorem 1.4. Suppose that $M$ is a closed, simple 3-manifold which contains a connected closed incompressible surface of genus 2 or 3 , and that $\operatorname{Hg}(M) \geq 8$. Then $M$ contains a connected closed incompressible surface $S$ of genus at most 4 , such that either $\bar{\chi}(\operatorname{kish}(M \backslash \backslash S)) \geq 2$, or $S$ is separating and $M \backslash \backslash S$ has an acylindrical component $N$ with $\operatorname{Hg}(N) \geq 7$.

Proof. Let $M$ satisfy the hypotheses of Theorem 1.4, and note that since $M$ is simple, it is $(2,2)$-small by definition. Suppose $M$ contains a connected closed incompressible surface of genus 2 . If $M$ is $(3,2)$-small, the hypothesis on $\operatorname{Hg}(M)$ and [6, Theorem 3.2] imply that for any such surface $S$, $\bar{\chi}(\operatorname{kish}(M \backslash \backslash S)) \geq 2$, satisfying the first conclusion of Theorem 1.4. Otherwise, Culler et al. [6, Theorem 5.8] provides a separating, connected, closed incompressible surface $S$ of genus 2 satisfying one of the conditions below:

(1) at least one component of $M \backslash \backslash S$ is acylindrical; or

(2) for each component $B$ of $M \backslash \backslash S$ we have $\operatorname{kish}(B) \neq \emptyset$. 
If $S$ satisfies condition (2), then since each component $B$ of $M \backslash \backslash S$ has kish $B \neq \emptyset$ we have $\bar{\chi}(\operatorname{kish}(M \backslash \backslash S)) \geq 2$, which implies the first conclusion of Theorem 1.4. We address the other case below.

Now suppose that $M$ contains no connected closed incompressible surface of genus 2 but contains a connected closed incompressible surface of genus 3. If $M$ is $(5,3)$-small, the hypothesis on $\operatorname{Hg}(M)$ and [6, Theorem 3.2] imply that for any such surface $S, \bar{\chi}(\operatorname{kish}(M \backslash \backslash S)) \geq 4$, satisfying the first conclusion of Theorem 1.4. The remaining possibilities are that $M$ contains a separating incompressible surface of genus $g$ and is $(g, 3)$-small, for $g=3$ or 4 . In either case, the hypothesis on Heegaard genus ensures that $[6$, Theorem 5.8] provides a separating connected closed incompressible surface $S$ of genus $g$ satisfying condition (1) or (2) above. As above, if $S$ satisfies conclusion (2), then the first conclusion of Theorem 1.4 follows.

In the remaining cases, we have a separating, connected, closed incompressible surface $S \subset M$ of genus 2, 3 or 4, satisfying condition (1) above, and we may assume that $S$ does not satisfy condition (2) there. Let $N$ be an acylindrical component of $M \backslash \backslash S$ and $B$ the remaining component. Since $N$ is acylindrical, $N=\operatorname{kish}(N)$. Therefore $\operatorname{kish}(B)=\emptyset$, since otherwise $S$ would satisfy condition (2) above. Then $B=|\mathcal{B}|$ for some book of $I$-bundles $\mathcal{B}$ (cf. $[6, \S 5.1]$ ), and so by Culler et al. [6, Lemma 5.3], $B$ is "shallow relative to $S$ " [6, Definition 4.3]. It now follows from [6, Lemma 4.4] that $\operatorname{Hg}(M) \leq 1+\operatorname{Hg}(N)$, hence by hypothesis that $\operatorname{Hg}(N) \geq 7$. Thus in this case the second conclusion of Theorem 1.4 holds.

Theorem 1.3. Let $M$ be a closed, orientable hyperbolic 3-manifold containing a closed, connected incompressible surface of genus 2 or 3 , and suppose that $\operatorname{Hg}(M) \geq 8$. Then $M$ has volume greater than 6.89 .

Proof. We apply Theorem 1.4 to $M$, yielding a connected closed surface $S$ of genus at most 4 satisfying its conclusion. If $\bar{\chi}(\operatorname{kish}(M \backslash \backslash S)) \geq 2$, then Theorem 9.1 of [2] implies that the volume of $M$ is greater than 7.32. Thus we assume $S$ is separating and $M \backslash \backslash S$ has an acylindrical component $X$ with $\operatorname{Hg}(X) \geq 7$. It is a standard result (cf. [6, Proposition 6.3]) that $X$ is homeomorphic to a hyperbolic 3 -manifold $N$ with totally geodesic boundary, and $\operatorname{vol}(N)=\operatorname{geod} \operatorname{vol}(X)$ (see Definition 6.2 of $[6]$ ).

If $S$ has genus at least 3, then by Miyamoto's Theorem [18, Theorem $5.4], \operatorname{vol}(N)>10.4$. If $S$ has genus 2 , then Theorem 1.1 implies that $N$ has volume greater than 6.89. Theorem 1.3 now follows from [6, Proposition 6.4] (which is in turn derived from results in [2]). 
Theorem 1.2. Let $M$ be a closed, orientable hyperbolic 3-manifold with

$$
\operatorname{dim}_{\mathbb{Z}_{2}} H_{1}\left(M ; \mathbb{Z}_{2}\right) \geq 5
$$

and suppose that the cup product map $H^{1}\left(M ; \mathbb{Z}_{2}\right) \otimes H^{1}\left(M ; \mathbb{Z}_{2}\right) \rightarrow H^{2}(M$; $\mathbb{Z}_{2}$ ) has image of dimension at most one. Then $M$ has volume greater than 3.44 .

Proof. If $\pi_{1} M$ is 4 -free, then Theorem 1.2 of [7] implies that $M$ has volume greater than 3.44. Otherwise there is a subgroup $G$ of $\pi_{1} M$ which has rank at most 4 and is not free. The homological hypotheses and Proposition 3.5 of [9] ensure that there is a two-fold cover $\widetilde{M} \rightarrow M$, with $\operatorname{dim}_{\mathbb{Z}_{2}} H_{1}\left(\widetilde{M} ; \mathbb{Z}_{2}\right) \geq 8$, such that $G<\pi_{1} \widetilde{M}$. Theorem 1.1 of [7] implies that $\widetilde{M}$ contains an incompressible surface of genus 2 or 3 . Since $\mathrm{Hg}(\widetilde{M})$ bounds above the dimension of its $\mathbb{Z}_{2}$-homology, we have $\operatorname{Hg}(\widetilde{M}) \geq 8$. Theorem 1.3 now implies that $\widetilde{M}$ has volume greater than 6.89 ; hence that $M$ has volume greater than 3.445 .

\section{Acknowledgments}

Partially supported by NSF grant DMS-0703749. Partially supported by NSF grants DMS-0204142 and DMS-0504975. The authors thank the referee for a thorough reading and helpful comments, and in particular for pointing out a gap in the original proof of Theorem 7.4 .

\section{References}

[1] I. Agol, M. Culler, and P.B. Shalen, Singular surfaces, mod 2 homology, and hyperbolic volume I, Trans. Amer. Math. Soc. 362 (2010), 3463-3498.

[2] I. Agol, P.A. Storm and W.P. Thurston, Lower bounds on volumes of hyperbolic Haken 3-manifolds, J. Amer. Math. Soc. 20(4) (2007), 10531077 (electronic). With an appendix by Nathan Dunfield.

[3] F. Bonahon, Cobordism of automorphisms of surfaces, Ann. Sci. École Norm. Sup. (4) 16(2) (1983), 237-270.

[4] K. Böröczky, Packing of spheres in spaces of constant curvature, Acta Math. Acad. Sci. Hungar. 32(3-4) (1978), 243-261.

[5] A.J. Casson and C. McA. Gordon, Reducing Heegaard splittings, Topol. Appl. 27(3) (1987), 275-283. 
[6] M. Culler, J. DeBlois, and P.B. Shalen, Incompressible surfaces, hyperbolic volume, Heegaard genus and homology, Comm. Anal. Geom. 17(2) (2009), 155-184.

[7] M. Culler and P. Shalen, 4-free groups and hyperbolic geometry, Preprint, arXiv:0806.1188v1, June 2008.

[8] M. Culler and P. Shalen, Singular surfaces, mod 2 homology, and hyperbolic volume, II, Preprint. arXiv:math/0701666v5, May 2008.

[9] Marc Culler and P.B. Shalen, Volume and homology of one-cusped hyperbolic 3-manifolds, Algebr. Geom. Topol. 8(1) (2008), 343-379.

[10] D.B.A. Epstein, Curves on 2-manifolds and isotopies, Acta Math. 115 (1966) 83-107.

[11] M. Freedman, J. Hass and P. Scott, Closed geodesics on surfaces, Bull. London Math. Soc. 14(5) (1982), 385-391.

[12] R. Frigerio, B. Martelli and C. Petronio, Small hyperbolic 3-manifolds with geodesic boundary, Experiment. Math. 13(2) (2004), 171-184.

[13] D. Gabai, R. Meyerhoff and P. Milley, Mom technology and volumes of hyperbolic 3-manifolds, Preprint, arXiv:math/0606072, July 2006.

[14] W.H. Jaco and P.B. Shalen, Seifert fibered spaces in 3-manifolds, Mem. Amer. Math. Soc. 21(220) (1979), viii+192.

[15] K. Johannson, Homotopy equivalences of 3-manifolds with boundaries, Lect. Notes in Math., 761, Springer, Berlin, 1979.

[16] S. Kojima, Polyhedral decomposition of hyperbolic 3-manifolds with totally geodesic boundary, in Aspects of Low-dimensional Manifolds, Adv. Stud. Pure Math., 20, Kinokuniya, Tokyo, 1992, 93-112.

[17] S. Kojima and Y. Miyamoto, The smallest hyperbolic 3-manifolds with totally geodesic boundary, J. Differential Geom. 34(1) (1991), 175-192.

[18] Y. Miyamoto, Volumes of hyperbolic manifolds with geodesic boundary, Topology 33(4) (1994), 613-629.

[19] J.G. Ratcliffe, Foundations of hyperbolic manifolds, Graduate Texts Math., 149, Springer, New York, 1994.

[20] J. Schultens, The classification of Heegaard splittings for (compact orientable surface) $\times S^{1}$, Proc. London Math. Soc. (3) 67(2) (1993), 425-448. 
[21] F. Waldhausen, On irreducible 3-manifolds which are sufficiently large, Ann. of Math. (2) 87 (1968), 56-88.

Department of Mathematics

Statistics, and Computer Science (M/C 249)

University of Illinois at CHICAGO

851 S. Morgan Street

Chicago, IL 60607-7045

USA

E-mail address: jdeblois@math.uic.edu; shalen@math.uic.edu

Received Month 00, 2010 
